\title{
GLOBAL TOPOLOGICAL CONFIGURATIONS OF SINGULARITIES FOR THE WHOLE FAMILY OF QUADRATIC DIFFERENTIAL SYSTEMS
}

\author{
JOAN C. ARTÉS ${ }^{1}$, JAUME LLIBRE $^{1}$, DANA SCHLOMIUK ${ }^{2}$ AND NICOLAE VULPE ${ }^{3}$
}

\begin{abstract}
In [1] the authors proved that there are 1765 different global geometrical configurations of singularities of quadratic differential systems in the plane. There are other 8 configurations conjectured impossible, all of them related with a single configuration of finite singularities. This classification is completely algebraic and done in terms of invariant polynomials and it is finer than the classification of quadratic systems according to the topological classification of the global configurations of singularities, the goal of this article. The long term project is the classification of phase portraits of all quadratic systems under topological equivalence. A first step in this direction is to obtain the classification of quadratic systems under topological equivalence of local phase portraits around singularities. In this paper we extract the local topological information around all singularities from the 1765 geometric equivalence classes. We prove that there are exactly 208 topologically distinct global topological configurations of singularities for the whole quadratic class. The 8 global geometrical configurations conjectured impossible do not affect this number of 208. From here the next goal would be to obtain a bound for the number of possible different phase portraits, modulo limit cycles.
\end{abstract}

\section{INTRODUCTION AND STATEMENT OF MAIN RESULTS}

We consider here differential systems of the form

$$
\frac{d x}{d t}=p(x, y), \quad \frac{d y}{d t}=q(x, y),
$$

where $p, q \in \mathbb{R}[x, y]$, i.e. $p, q$ are polynomials in $x, y$ over $\mathbb{R}$. We call degree of a system (1) the integer $m=\max (\operatorname{deg} p, \operatorname{deg} q)$. In particular we call quadratic a differential system (1) with $m=2$. We denote here by QS the whole class of real quadratic differential systems.

Polynomial systems (1) intervene in many areas of applied mathematics. They are also interesting from the theoretical viewpoint since some problems stated over 100 years ago are still unsolved even for the quadratic class. Hilbert's 16th problem asks to determine for each positive integer $n$ the maximum number of limit cycle which a system (1) of degree $n$ could have, in case there is a finite bound for the number of limit cycles of such systems. So far, not even the finiteness part of Hilbert's 16'th problem was proved and this not even for quadratic systems. The finiteness part of Hilbert's 16th problem asks for a proof that for every positive integer $n$ there exists an integer $N$ such that every system (1) of degree $n$ has at most $N$ limit cycles. These are not the only longstanding open problems on systems (1).

There is a large number of papers on quadratic systems. For early short surveys on quadratic systems see $[5,4]$. A more recent account on quadratic systems is given in [9] which contains many phase portraits of quadratic systems, the harder cases being left open. The most recent and most complete survey on quadratic systems is given in the book [1] where the family QS is classified according to the geometric equivalence relation for configurations of singularities of the systems. In this book it was proved that there are at least 1765 and at most 1773 such geometric configurations of singularities for quadratic differential systems.

1991 Mathematics Subject Classification. Primary 58K45, 34C05, 34A34.

Key words and phrases. quadratic vector fields, infinite and finite singularities, affine invariant polynomials, Poincaré compactification, configuration of singularities, topological equivalence relation.

The first two authors are partially supported by the Ministerio de Economia, Industria y Competitividad, Agencia Estatal de Investigación grant MTM2016-77278-P (FEDER), the Agència de Gestió d'Ajuts Universitaris i de Recerca grant 2017 SGR 1617, and the European project Dynamics-H2020-MSCA-RISE-2017-777911. The third author is supported by NSERC. The fourth author is supported by the grant 12.839.08.05F from SCSTD of ASM and partially by NSERC. 
The long term goal of our program is to classify QS according to the topological equivalence relation of phase portraits modulo limit cycles, concept defined below. It is well known (see [5]) that any limit cycle of a quadratic system surrounds exactly one singularity which is a focus.

By topological equivalence relation modulo limit cycles (of quadratic systems) we mean the following: two systems $S_{1}, S_{2}$ are equivalent modulo limit cycles $S_{1} \sim_{L C} S_{2}$, if and only if by identifying the unique focus inside each eye of limit cycles with each one of the points inside the closed region bounded by the largest one of the limit cycles of an eye of limit cycles, the two phase portraits become topologically equivalent with the resulting quotient topology on the plane. Then the classification problem modulo limit cycles is the problem of describing the complete list of phase portraits of the quadratic class obtained in this way, i.e. one phase portrait for each equivalence class.

In case the systems were of higher degree, the equivalence modulo limit cycles should be redefined so as to cover other possibilities such as having several singularities inside the limit cycles, or even several nests of limit cycles inside a bigger one.

To obtain the topological classification modulo limit cycles we need to start with the study of the topological configurations of singularities.

Definition 1. By topological configuration of singularities of a system (1) we mean an ordered couple whose first component is the list of local phase portraits around finite singularities. The second component is the list of phase portraits around infinite singularities, endowed with an order (clockwise or counterclockwise) taking into account the relative position of the sectors with respect to the line at infinity.

Definition 2. (i) Let $\chi_{1}$ and $\chi_{2}$ be two non-degenerate polynomial differential systems with a finite number of singularities, finite or infinite. We say that $\chi_{1}$ and $\chi_{2}$ have topologically equivalent configurations of singularities if and only if we have a bijection $\sigma$ of the set of singularities of $\chi_{1}$ onto the set of singularities of $\chi_{2}$ (sending finite singularities to finite singularities and infinite ones to infinite ones) such that for every singularity $s$ of $\chi_{1}, \sigma(s)$ is topologically equivalent to $s$ and furthermore the restriction $\sigma_{\infty}$ to the subset of infinite singularities listed in anyone of the two directions (clockwise or counterclockwise) on the Poincaré disk maintains the relative position of the sectors with respect to the line at infinity.

(ii) Let $\chi_{1}$ and $\chi_{2}$ be two non-degenerate polynomial differential systems with a finite number of finite singularities and an infinite number of infinite singularities. We say that the configurations of the two systems are topologically equivalent if the configurations of the finite singularities are topologically equivalent and the configurations of singularities which appear from the reduction obtained after the removal of the line at infinity in the two charts, are geometrically equivalent.

(iii) Let $\chi_{1}$ and $\chi_{2}$ be two degenerate quadratic differential systems. We say that the configurations of these two systems are topologically equivalent if (a) there is a one-to-one correspondence $\sigma$ of the set of isolated singularities of $\chi_{1}$ onto the set of isolated singularities of $\chi_{2}$ such that for every isolated singularity $s, \sigma(s)$ and $s$ have topologically equivalent phase portraits around $\sigma(s)$ and $s$, and $(b)$ the two curves $f_{1}=0$ and $f_{2}=0$ where $f_{1}$ is the greatest common divisor over $\mathbb{R}$ of the two polynomials defining $\chi_{1}$, and $f_{2}$ is the greatest common divisor over $\mathbb{R}$ of the two polynomials defining $\chi_{2}$, are affinely equivalent. Moreover, the singularities which appear from the reduction after the removal of the line of degeneracy, must also be geometrically equivalent.

For the definition of geometrical equivalence see [1].

Remark 1. We point out the importance of the geometrical equivalence required for the singularities that appear after the removal of the curves of singularities (whether finite or infinite) since this affects the topological phase portraits around singularities. Moreover, a topological classification of configurations of singularities could not have been completely obtained prior to the geometrical classification. 
The equivalence relation of topological configurations of singularities yields a partition of QS, i.e. two systems are in the same class if and only if they have equivalent topological configurations of singularities.

To obtain the topological classification of quadratic systems modulo limit cycles, we must go a step further and obtain a complete study of all possible connections occurring in the family QS. This step will yield a finer partition of QS.

The goal of this paper is to provide a complete classification of QS according to the equivalence relation of topological configurations of singularities.

In order to achieve this goal we use the classification of QS according to the equivalence relation of geometrical configurations of singularities obtained in [1]. This equivalence relation is finer than the equivalence relation of topological configurations of singularities, including besides local topological phase portraits, many other features, algebraic or geometric, such as for example orders of weak singularities, multiplicities, and tangential or blow-up equivalence. Our main result is the following one:

Main Theorem. (A) We consider here all configurations of singularities, finite and infinite, of quadratic vector fields. These configurations are classified in Diagrams 1-6 according to the topological equivalence relation of configurations. We have a total of 208 topologically distinct configurations of singularities, finite and infinite.

(B) Necessary and sufficient conditions for each one of the 208 different topological equivalence classes, can be assembled from these diagrams in terms of 41 invariant polynomials with respect to the action of the affine group and time rescaling. These invariant polynomials, which appear in the Diagrams 1-6, are given in Section 3.

(C) The Diagrams 1-6 actually contain the global bifurcation diagram in the 12-dimensional space of parameters, of the topological configurations of singularities, finite and infinite, of the family of quadratic differential systems and provide an algorithm for finding for any given quadratic differential system, its respective topological configuration of singularities.

There is already a work in progress concerning the 208 distinct topological configurations of singularities. More than 170 configurations have already been studied and more than 800 distinct phase portraits have already been obtained.

\section{Definitions And Notations for singularities of POLYNOMial Differential Systems}

In the book [1] we defined some new concepts for singularities closely related to the Jacobian matrix since they were more convenient for the geometrical classification than the classical concepts. The new definitions are:

We call elemental a singular point with its two eigenvalues not zero.

We call semi-elemental a singular point with exactly one of its eigenvalues equal to zero.

We maintain the name of nilpotent for a singular point with its two eigenvalues zero but with its Jacobian matrix at this point not identically zero.

We call intricate a singular point with its Jacobian matrix identically zero.

We defined notations for geometric configurations of singularities in [1]. Here we have to adapt and simplify for the specific case of topological configuration this notation. Some concepts such as for example order of weak singularities are not needed so we remove them. Even though other geometrical concepts like multiplicity or number of characteristic directions of nodes may seem irrelevant from the topological viewpoint, in some situations, in combinations with other phenomena, they provide topological difference in the local phase portraits.

First we start describing the finite and infinite singularities, denoting the first ones with lower case letters and the second with capital letters. When describing in a sequence both finite and infinite singular points, 


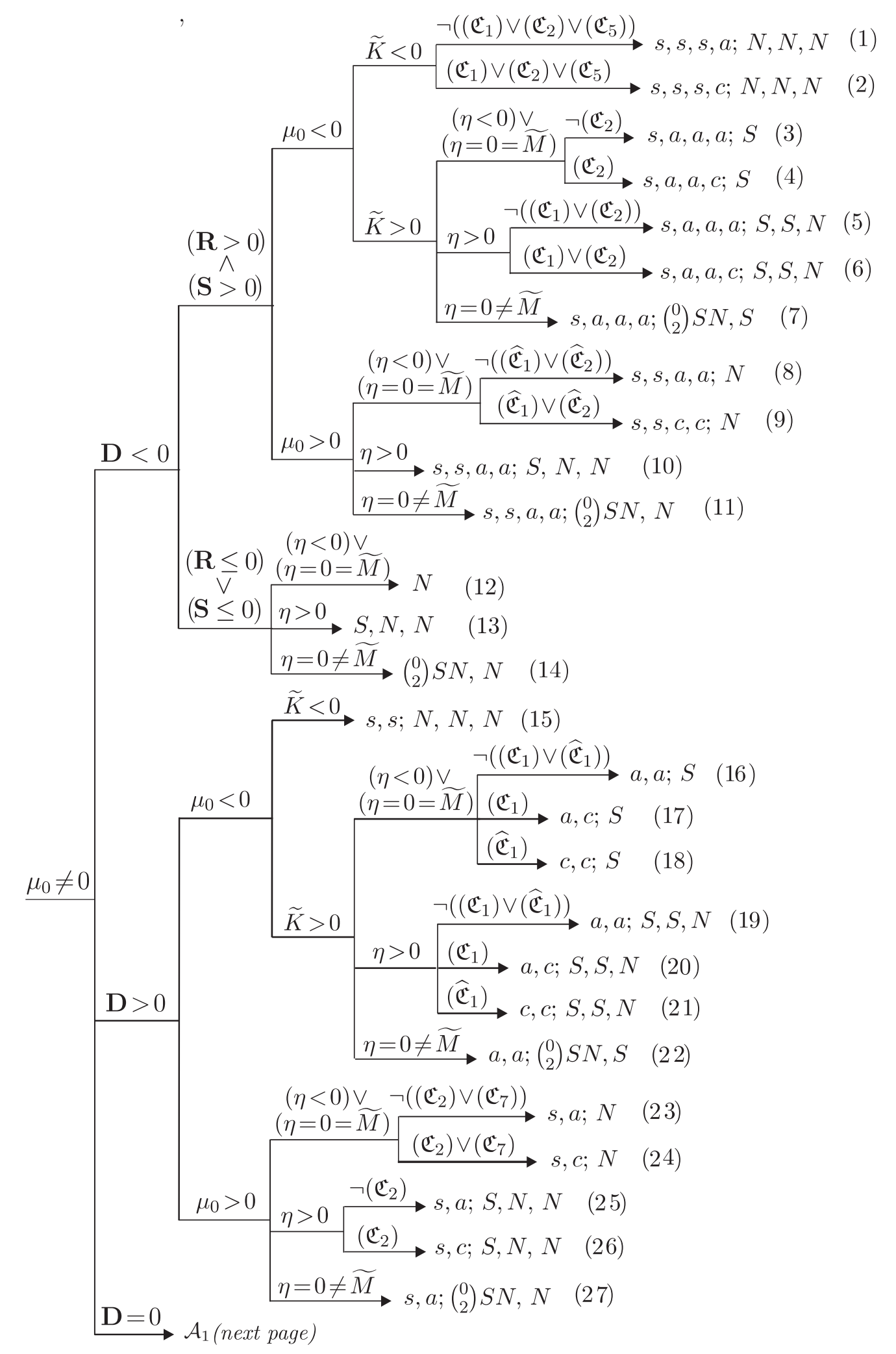

DiAGRAM 1. Bifurcation Diagram of global topological configurations: case of total multiplicity of finite singularities $m_{f}=4\left(\mu_{0} \neq 0\right)$. 


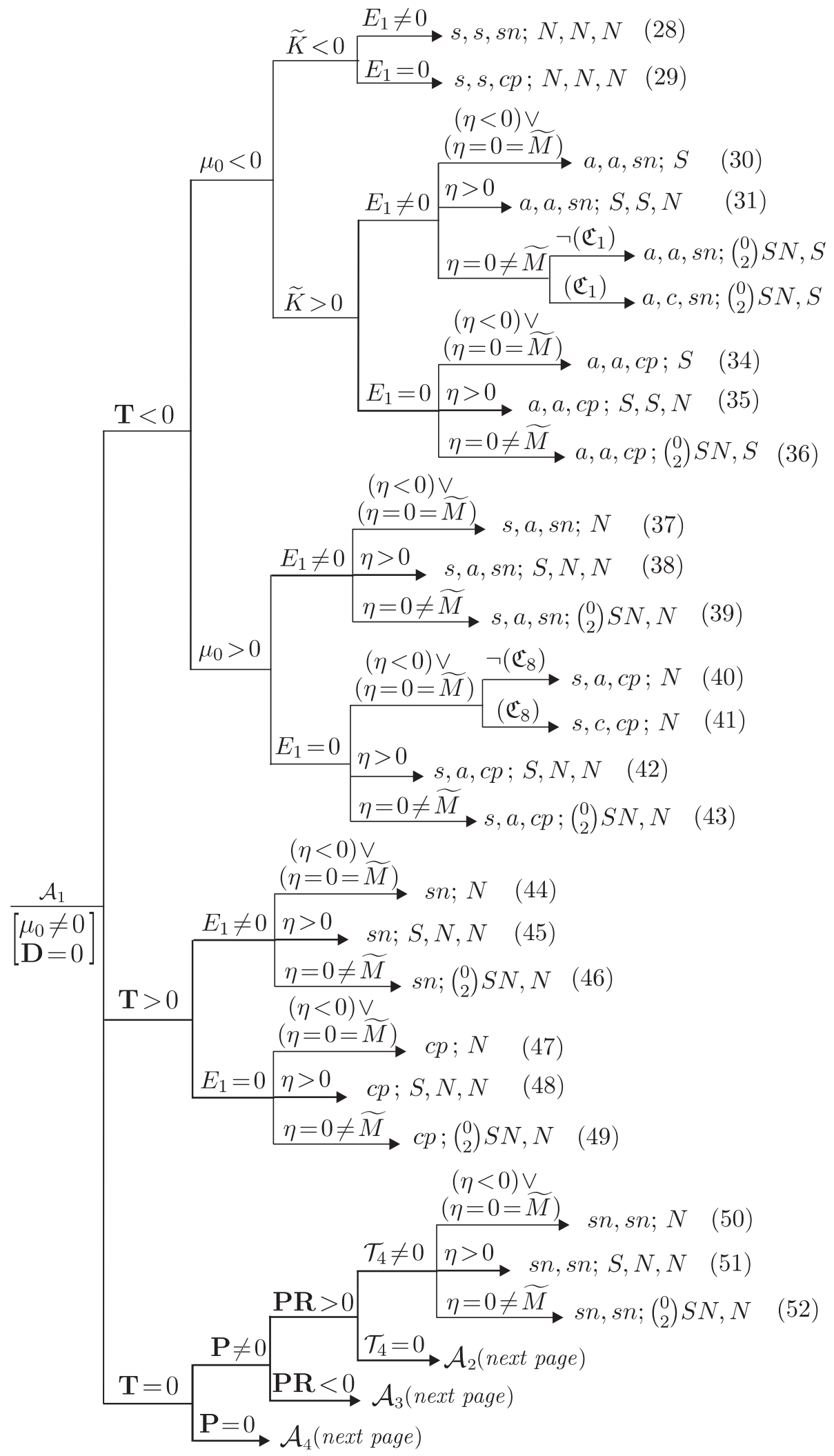
multiplicity of finite singularities $m_{f}=4\left(\mu_{0} \neq 0\right)$. 


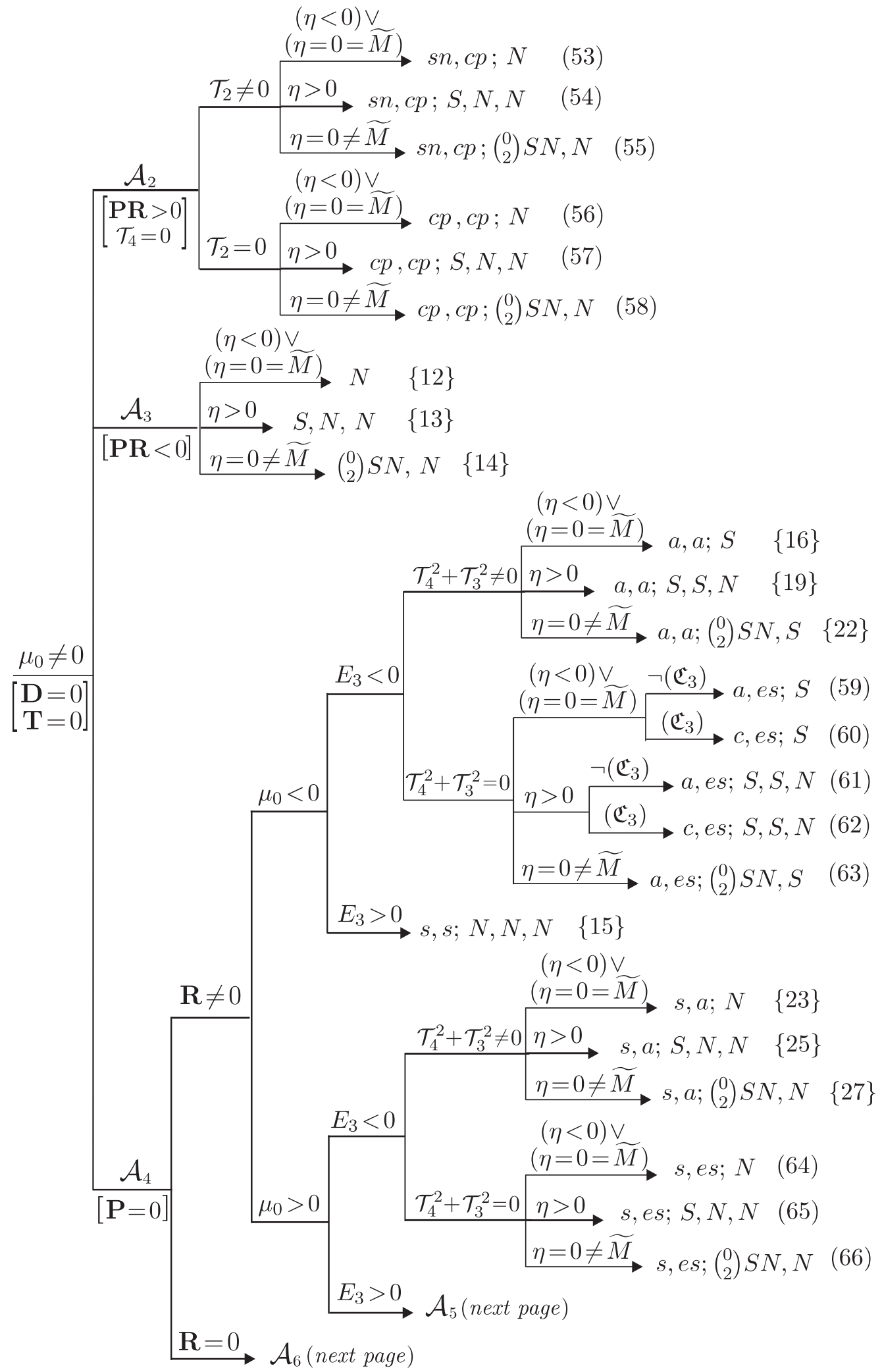

Diagram 1 (cont.): Bifurcation Diagram of global topological configurations: case of total multiplicity of finite singularities $m_{f}=4\left(\mu_{0} \neq 0\right)$. 


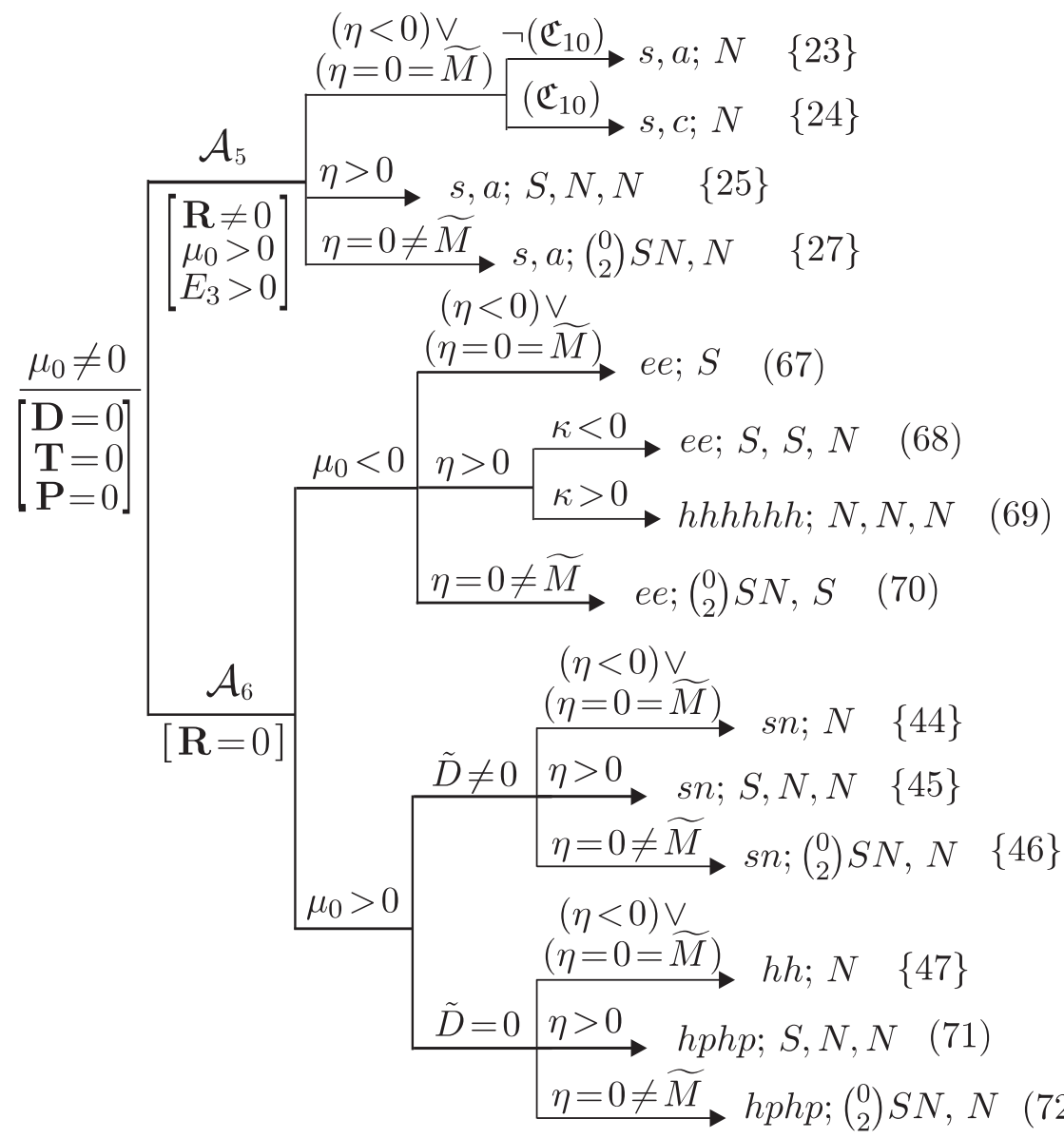

\section{Diagram 1 (cont.): Bifurcation Diagram of global topological configurations: case of total multiplicity of finite singularities $m_{f}=4\left(\mu_{0} \neq 0\right)$.}

we will always place first the finite ones and only later the infinite ones, separating them by a semicolon ';' . Even though finite and infinite singular points may either be real of complex, for the topological viewpoint, only the real ones are interesting and only these will be listed.

Elemental singularities: We use the letters ' $s$, ' $S$ ' for "saddles"; ' $n$ ', ' $N$ ' for "nodes"; ' $f$ ' for "foci" and ' $c$ ' for "centers". We will also denote by ' $a$ ' (anti-saddle) for either a focus or any type of node when the local phase portraits are topologically equivalent. Even though the number of characteristic direction of nodes are irrelevant from the topological viewpoint we need to keep these notations for the case they may be found under a line of singularities:

- ' $n$ ' for a node with two distinct eigenvalues;

- ' $n$ ' (a one-direction node) for a node with two identical eigenvalues whose Jacobian matrix cannot be diagonal;

- ' $n^{*}$ ' (a star node) for a node with two identical eigenvalues whose Jacobian matrix is diagonal.

Moreover, in the case of an elemental infinite node, there is a geometrical feature that distinguishes whether all orbits except one arrive tangent to infinity or to an affine direction. Even though this is irrelevant from the topological viewpoint in the case of isolated infinite singularities, it is not in case of some degenerate systems.

So we must use the notations ' $N$ ' and ' $N$ f for infinite nodes as they were defined in [1].

All non-elemental singular points are multiple points. Even though multiplicity is in most cases irrelevant for the local topological phase portrait, for some infinite singularities the type of multiplicity could be relevant and 


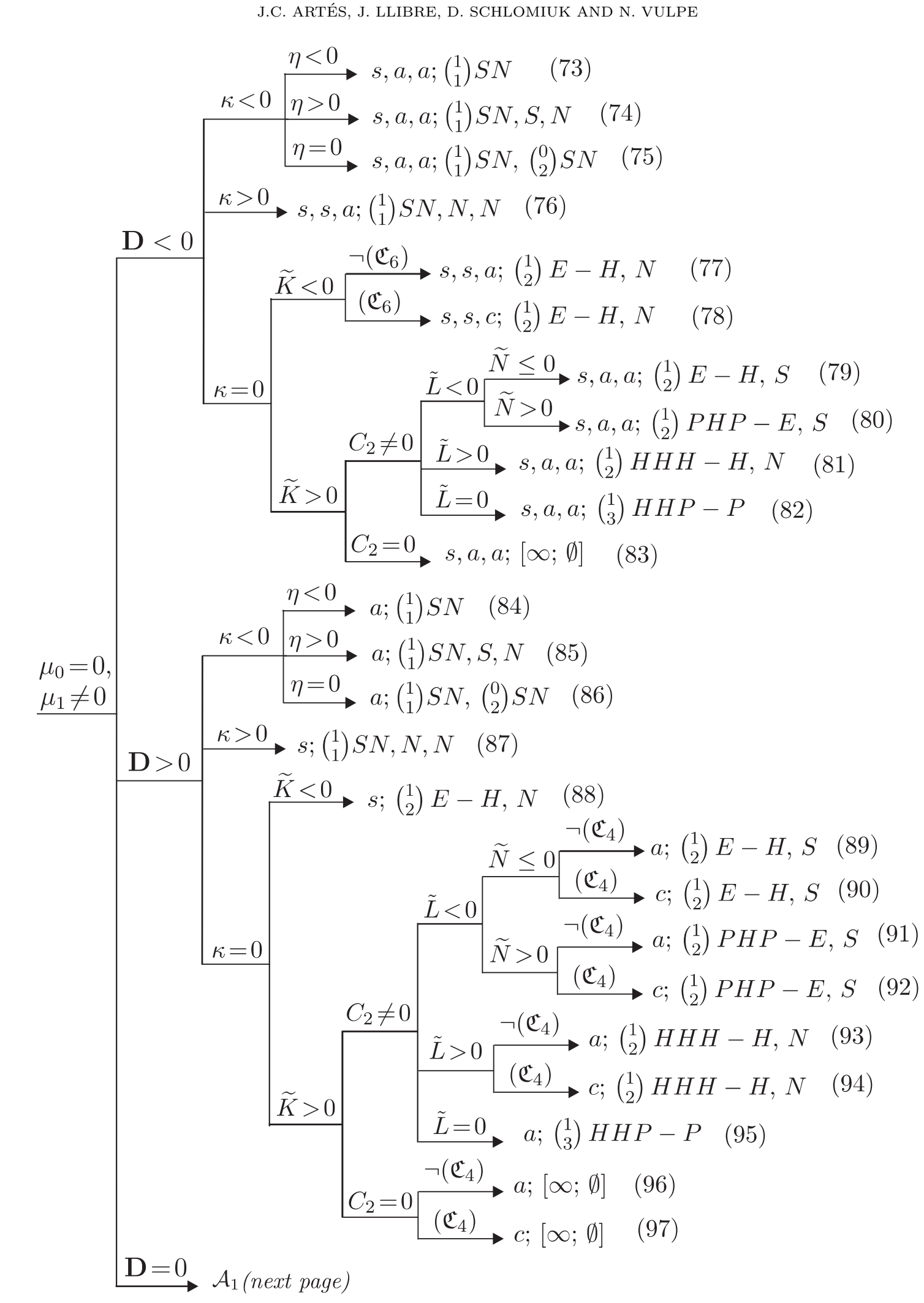

DIAGRAM 2. Bifurcation Diagram of global topological configurations: case of total multiplicity of finite singularities $m_{f}=3\left(\mu_{0}=0, \mu_{1} \neq 0\right)$. 


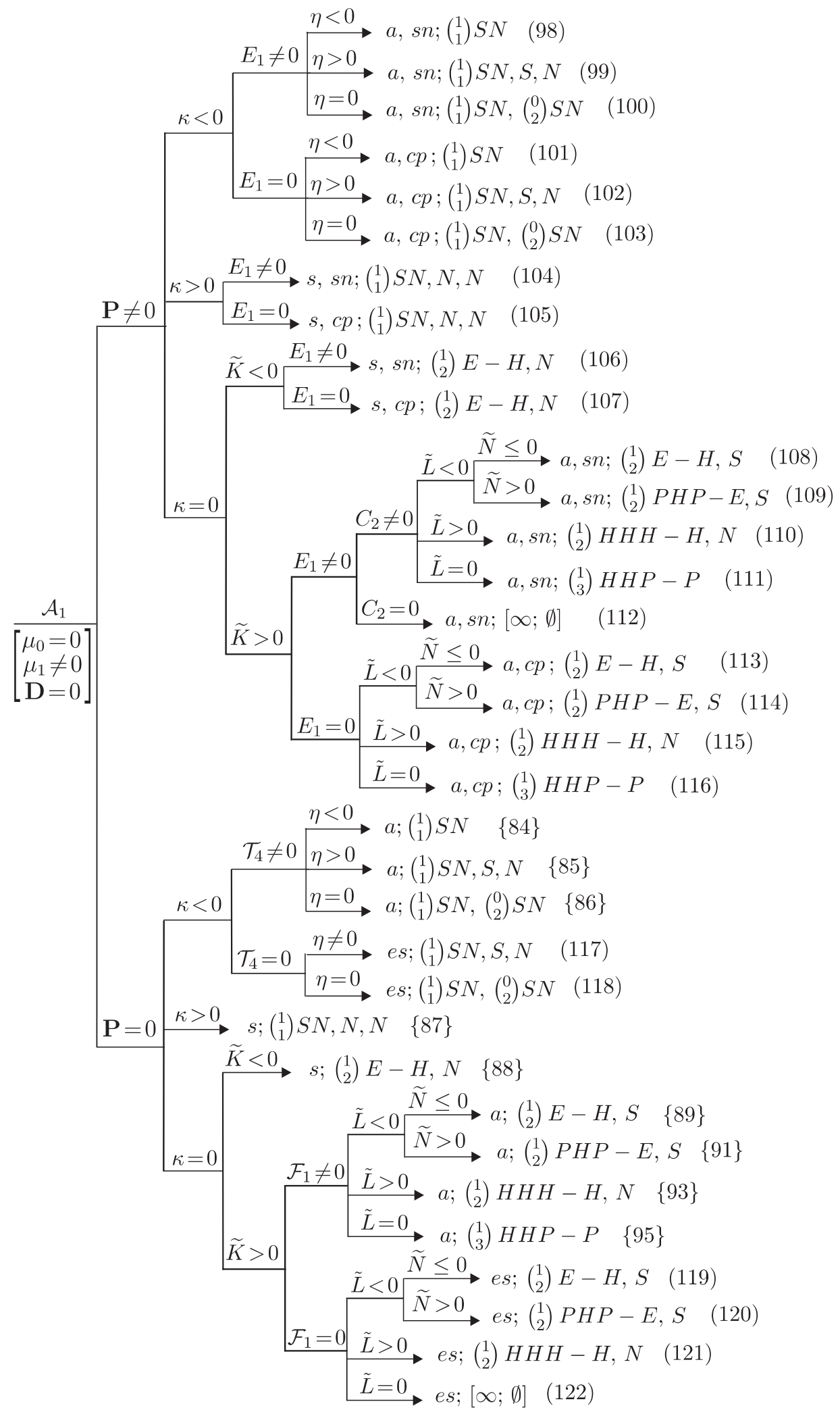
multiplicity of finite singularities $m_{f}=3\left(\mu_{0}=0, \mu_{1} \neq 0\right)$. 


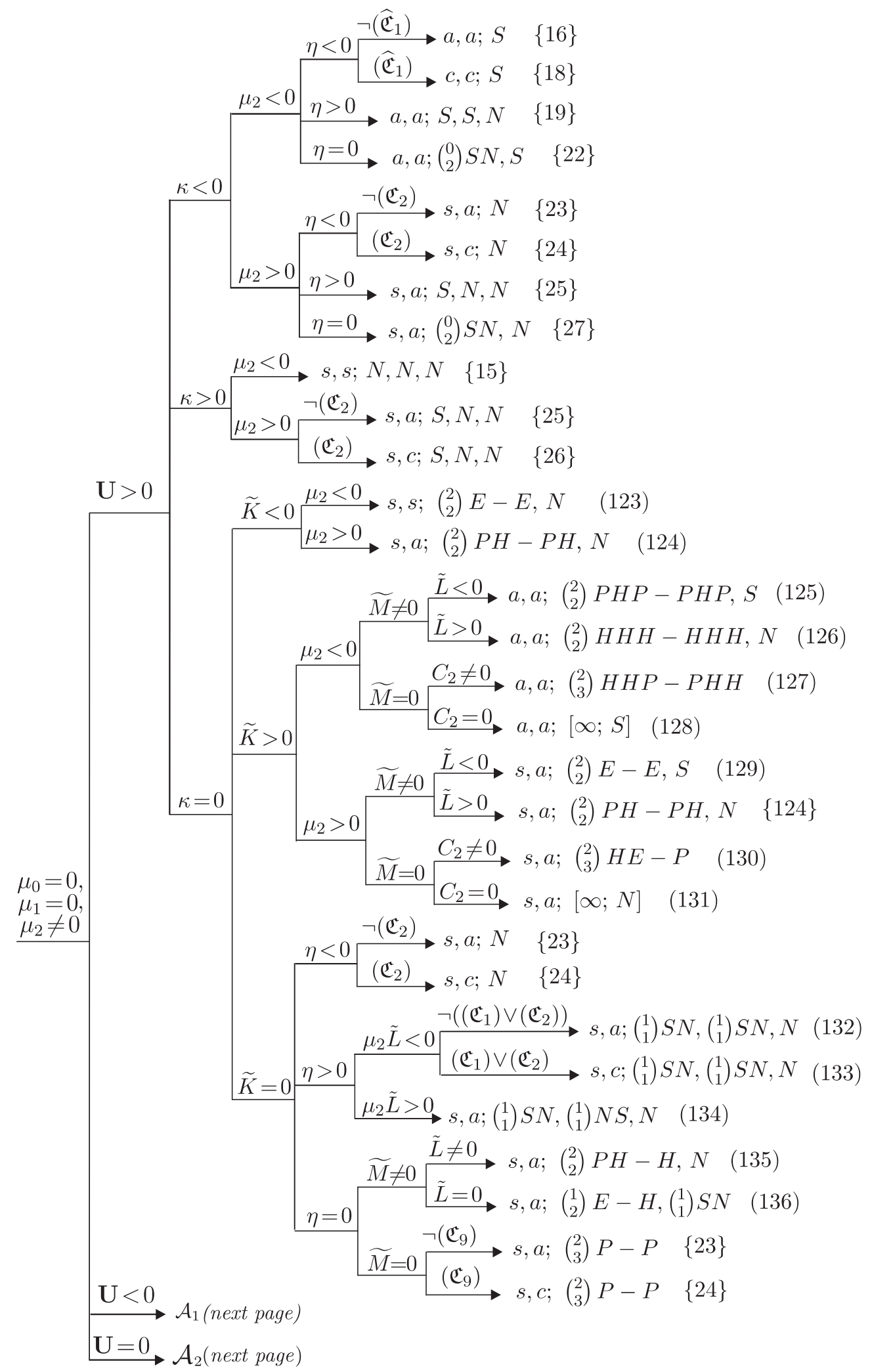

DiAGRAM 3. Bifurcation Diagram of global topological configurations: case of total multiplicity of finite singularities $m_{f}=2\left(\mu_{0}=\mu_{1}=0, \mu_{2} \neq 0\right)$. 


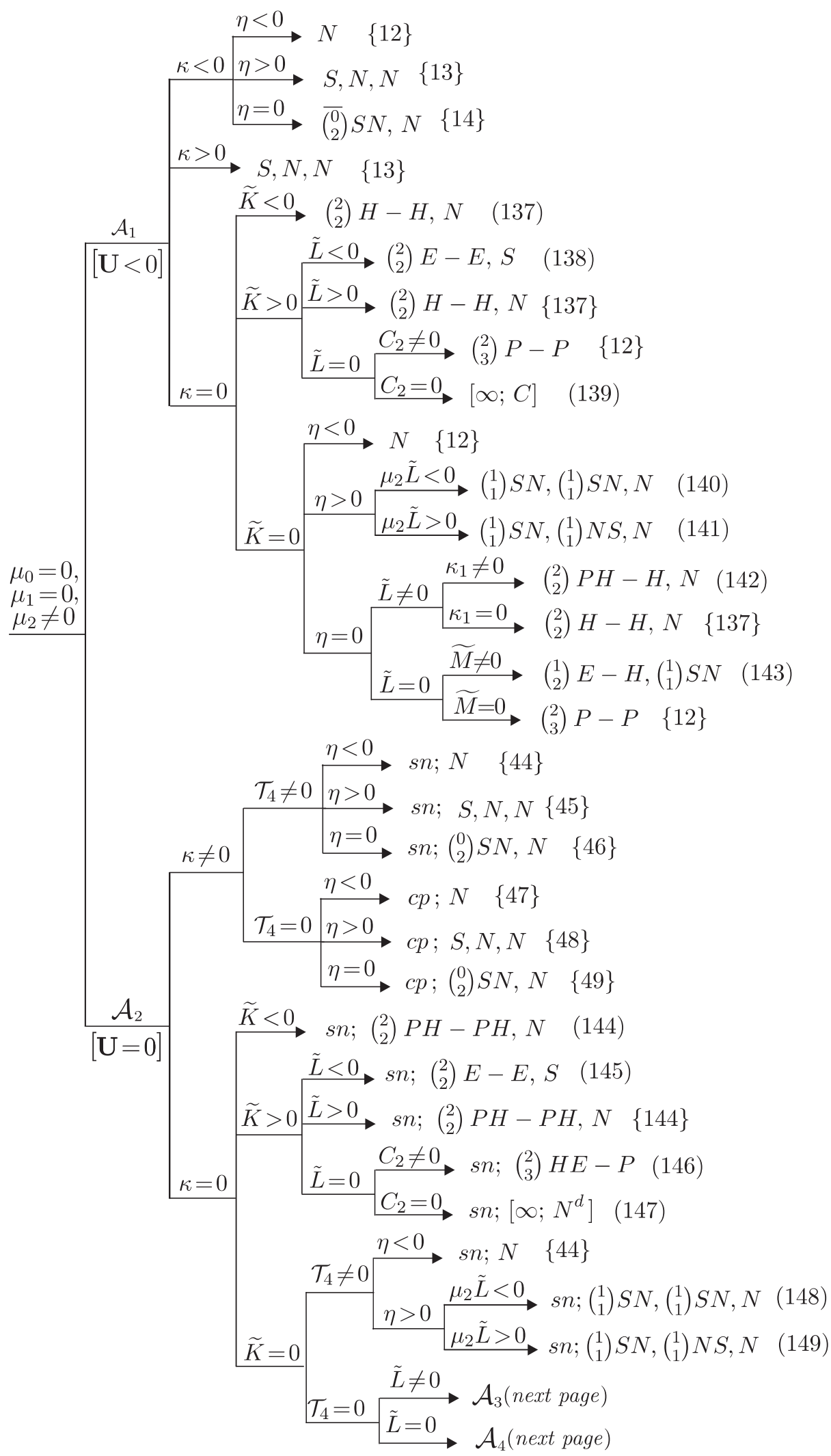

Diagram 3 (cont.): Bifurcation Diagram of global topological configurations: case of total multiplicity of finite singularities $m_{f}=2\left(\mu_{0}=\mu_{1}=0, \mu_{2} \neq 0\right)$. 


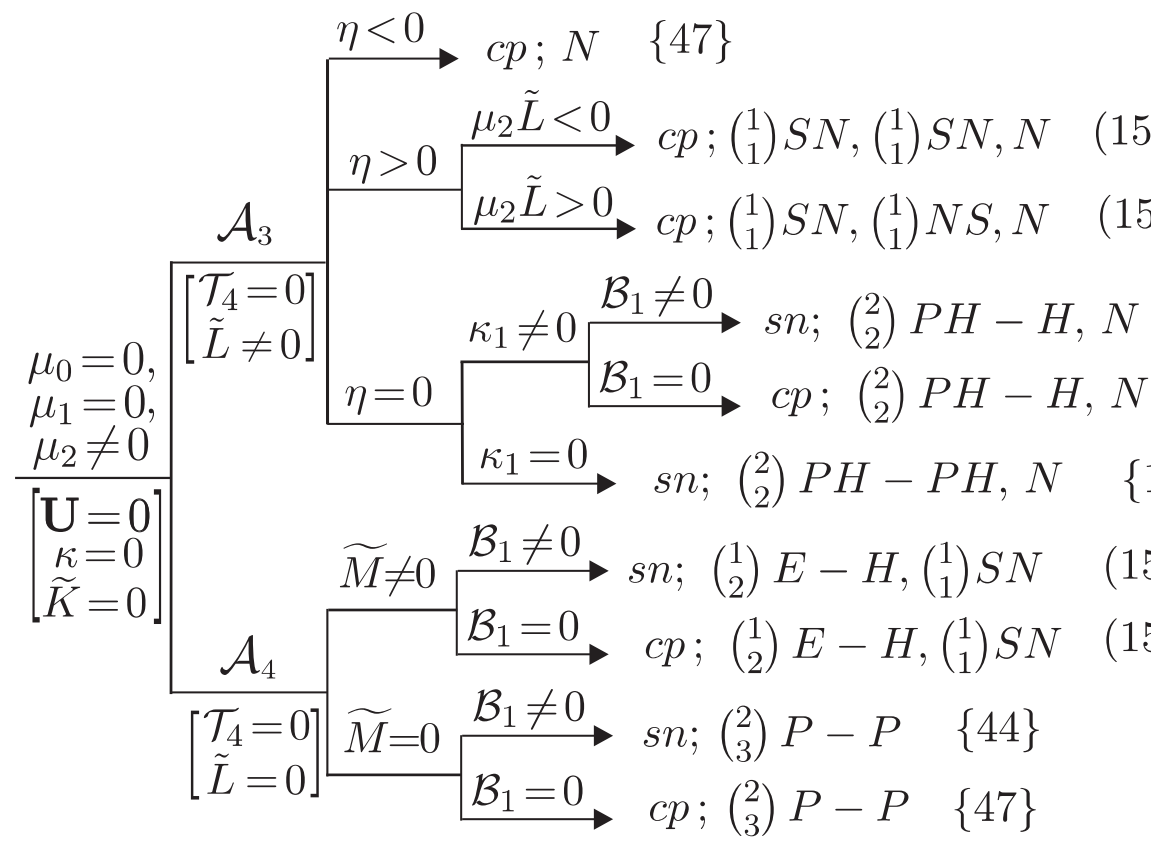

Diagram 3 (cont.): Bifurcation Diagram of global topological configurations: case of total multiplicity of finite singularities $m_{f}=2\left(\mu_{0}=\mu_{1}=0, \mu_{2} \neq 0\right)$.

we must point out the way we denote these cases. We denote by ' $\left(\begin{array}{l}a \\ b\end{array}\right)$...' the maximum number $a$ (respectively $b$ ) of finite (respectively infinite) singularities which can be obtained by perturbation of the multiple point. For example ' $\left(\begin{array}{l}1 \\ 1\end{array}\right) S N^{\prime}$ ' and $\left(\begin{array}{l}0 \\ 2\end{array}\right) S N^{\prime}$ ' correspond to two saddle-nodes at infinity which are locally topologically distinct.

Semi-elemental singularities: They can either be nodes, saddles or saddle-nodes, finite or infinite. However semi-elemental nodes and saddles are respectively topologically equivalent with elemental nodes and saddles. So we will use the same notation as if they were elemental ones. The only new semi-elemental singularity is the saddle-node which we denote by ' $s n$ '. As indicated above for infinite saddle-nodes $S N$ we will also keep the multiplicity. Moreover, as in [1] we also need the notation ' $\left(\begin{array}{l}1 \\ 1\end{array}\right) N S$ ' for some infinite saddle-nodes.

Nilpotent singularities: They can either be saddles, nodes, saddle-nodes, elliptic-saddles, cusps, foci or centers. The first four of these could be at infinity. The only finite nilpotent points for which we need to introduce notation are the elliptic-saddles and cusps which we denote respectively by $e s$ and $c p$.

In the case of nilpotent infinite points, the relative positions of the sectors with respect to the line at infinity, can produce topologically different phase portraits. This forces us to use a notation for these points similar to the notation which we will use for the intricate points.

Intricate singularities: It is known that the neighborhood of any singular point of a polynomial vector field (except for foci and centers) is formed by a finite number of sectors which could only be of three types: parabolic, hyperbolic and elliptic (see [6]). Then, a reasonable way to describe intricate and nilpotent points at infinity is to use a sequence formed by the types of their sectors. In the book [1] we use a geometrical notion of sector which is more subtle but which looses part of its meaning in the topological setting. More precisely any two adjacent parabolic geometrical sectors merge into one, and parabolic sectors adjacent to elliptic ones can be omitted. To lighten the notation, we make the convention to eliminate the parabolic sectors adjacent to the elliptic sectors. 


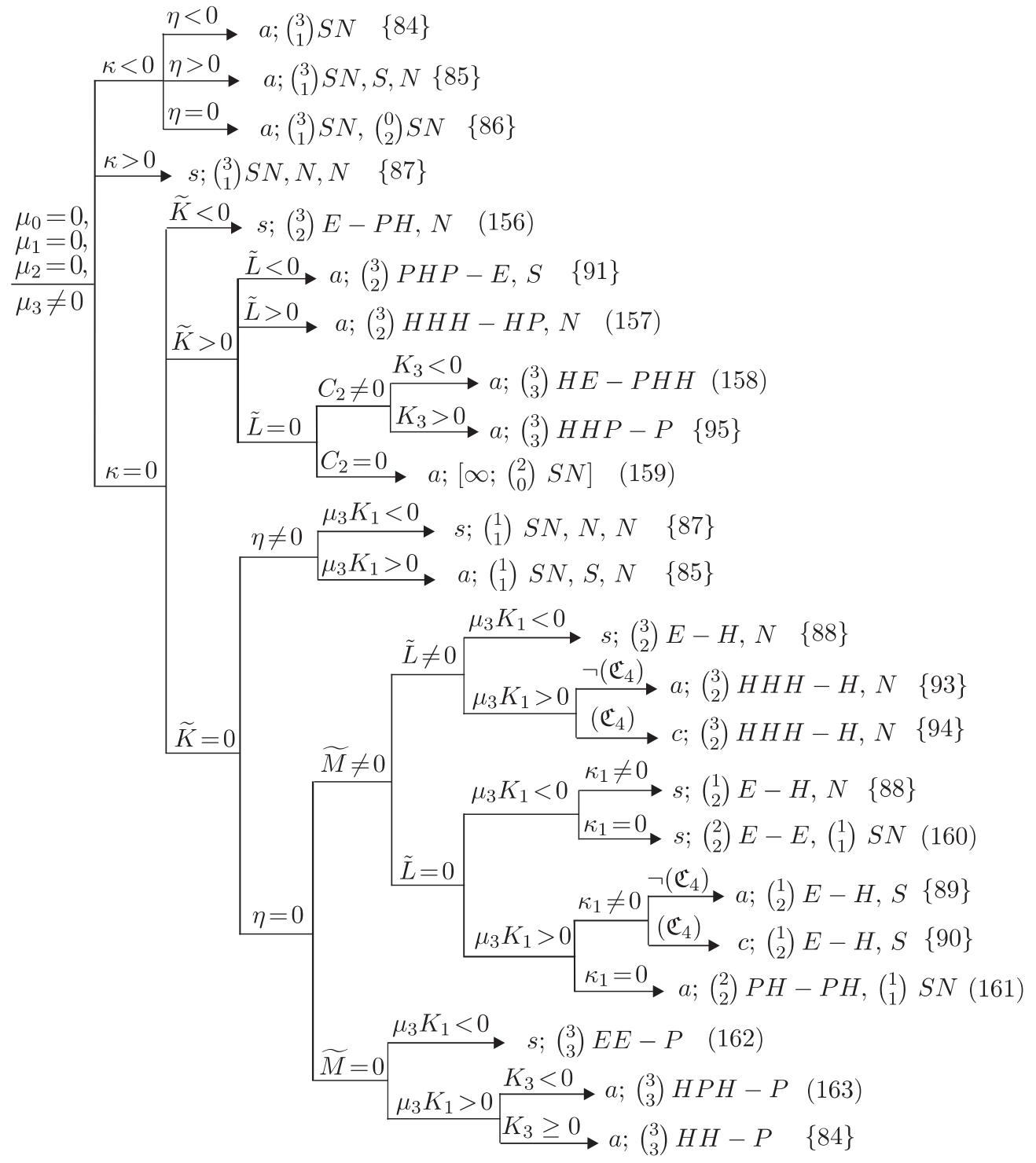

DiAgram 4. Bifurcation Diagram of global topological configurations: case of total multiplicity of finite singularities $m_{f}=1\left(\mu_{0}=\mu_{1}=\mu_{2}=0, \mu_{3} \neq 0\right)$.

Thus in quadratic systems, we have just four topological possibilities for finite intricate singular points of multiplicity four (see [2]) which are the following ones:

- a) $p h p h$;

- b) $h h$;

- c) $h h h h h h$;

- d) $e e$.

It is worth noting that the singularity $h h$ is topologically equivalent with $c p$. However we will keep the notation $h h$ for coherence with the rest of singularities.

For intricate and nilpotent singular points at infinity, we insert a dash (hyphen) between the sectors to split those which appear on one side or the other of the equator of the sphere. In this way we will distinguish between $\left(\begin{array}{l}2 \\ 2\end{array}\right) P-H H P$ and $\left(\begin{array}{l}2 \\ 2\end{array}\right) P H-H P$. When describing a single finite nilpotent or intricate singular point, one can always apply an affine change of coordinates to the system, so it does not really matter which sector 


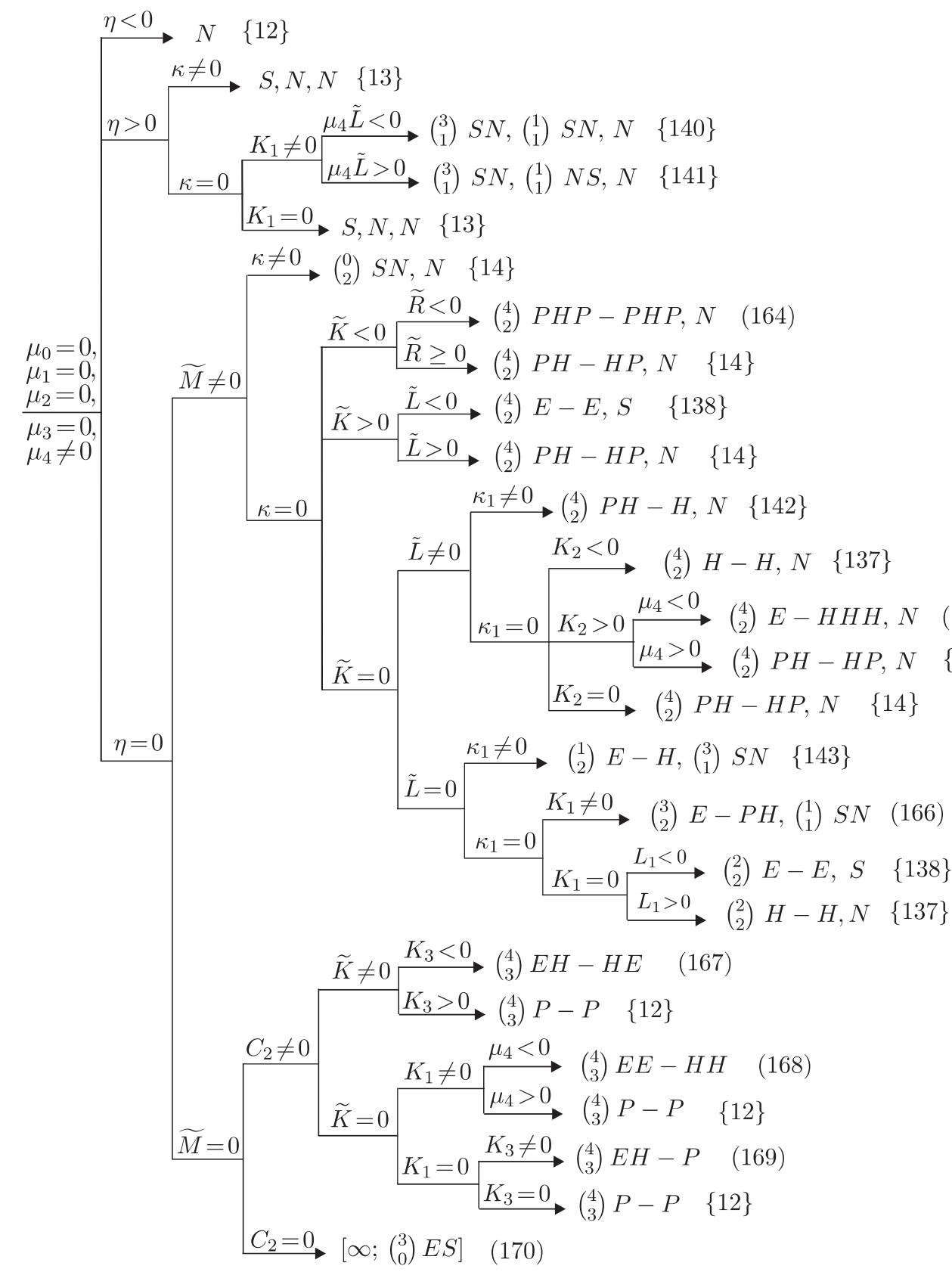

DiAgRAm 5. Bifurcation Diagram of global topological configurations: case of total multiplicity of finite singularities $m_{f}=0\left(\mu_{0}=\mu_{1}=\mu_{2}=\mu_{3}=0, \mu_{4} \neq 0\right)$.

starts the sequence, or the direction (clockwise or counter-clockwise) we choose. If it is an infinite nilpotent or intricate singular point, then we will always start with a sector bordering the infinity (to avoid using two dashes).

The lack of finite singular points after the removal of degeneracies, will be encapsulated in the notation $\emptyset$ (i.e. small size $\emptyset$ ). In similar cases when we need to point out the lack of an infinite singular point, we will use the symbol $\emptyset$.

Finally there is also the possibility that we have an infinite number of finite or of infinite singular points. In the first case, this means that the quadratic polynomials defining the differential system are not coprime. Their common factor may produce a line or conic with real coefficients filled up with singular points. 


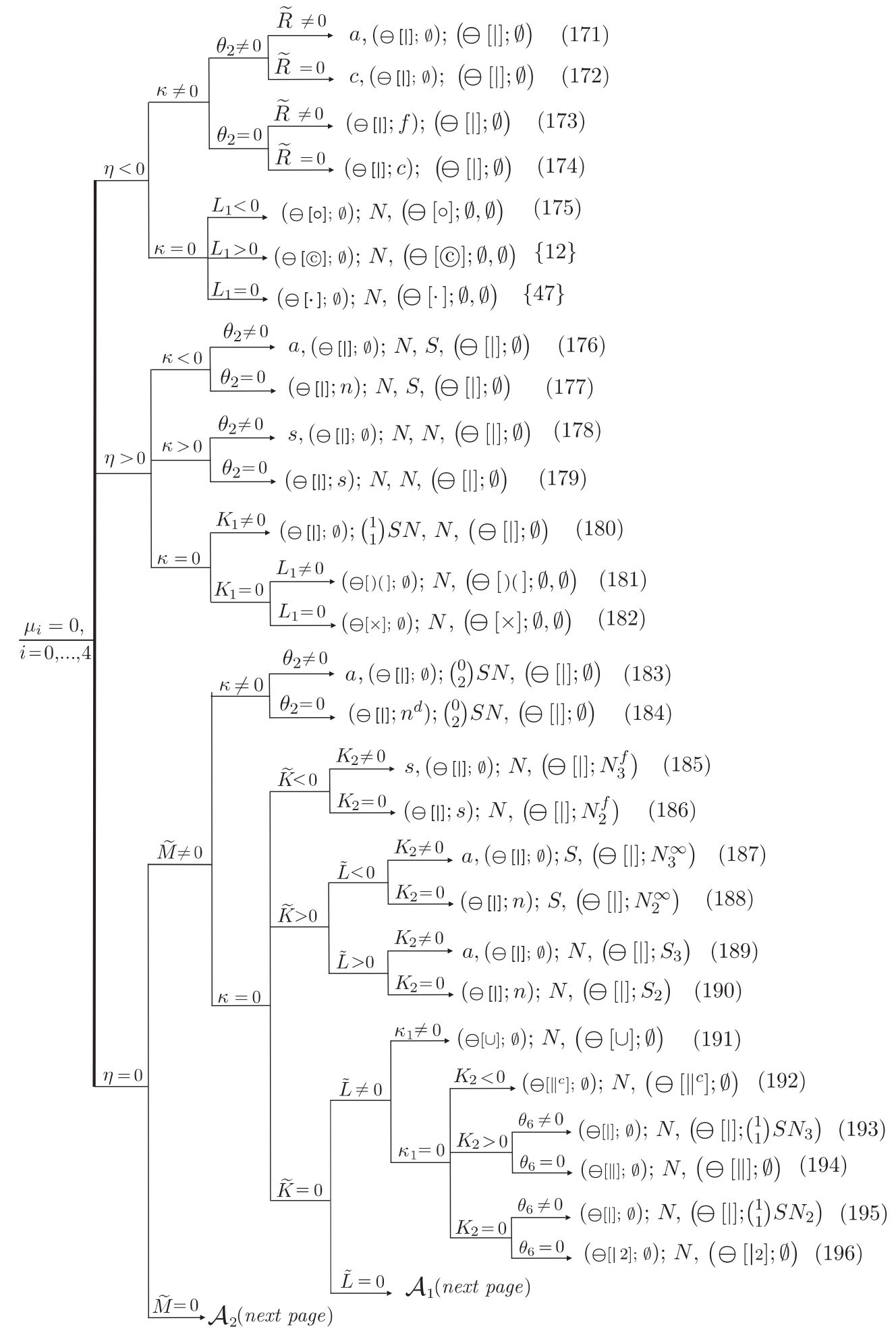

DiAgram 6. Bifurcation Diagram of global topological configurations: case of degenerate systems $\left(\mu_{0}=\mu_{1}=\mu_{2}=\mu_{3}=\mu_{4}=0\right)$.

Line at infinity filled up with singularities: It is known that any such system has in a sufficiently small neighborhood of infinity one of 6 topological distinct phase portraits (see [10]). The way to determine these portraits is by studying the reduced systems on the infinite local charts after removing the degeneracy 


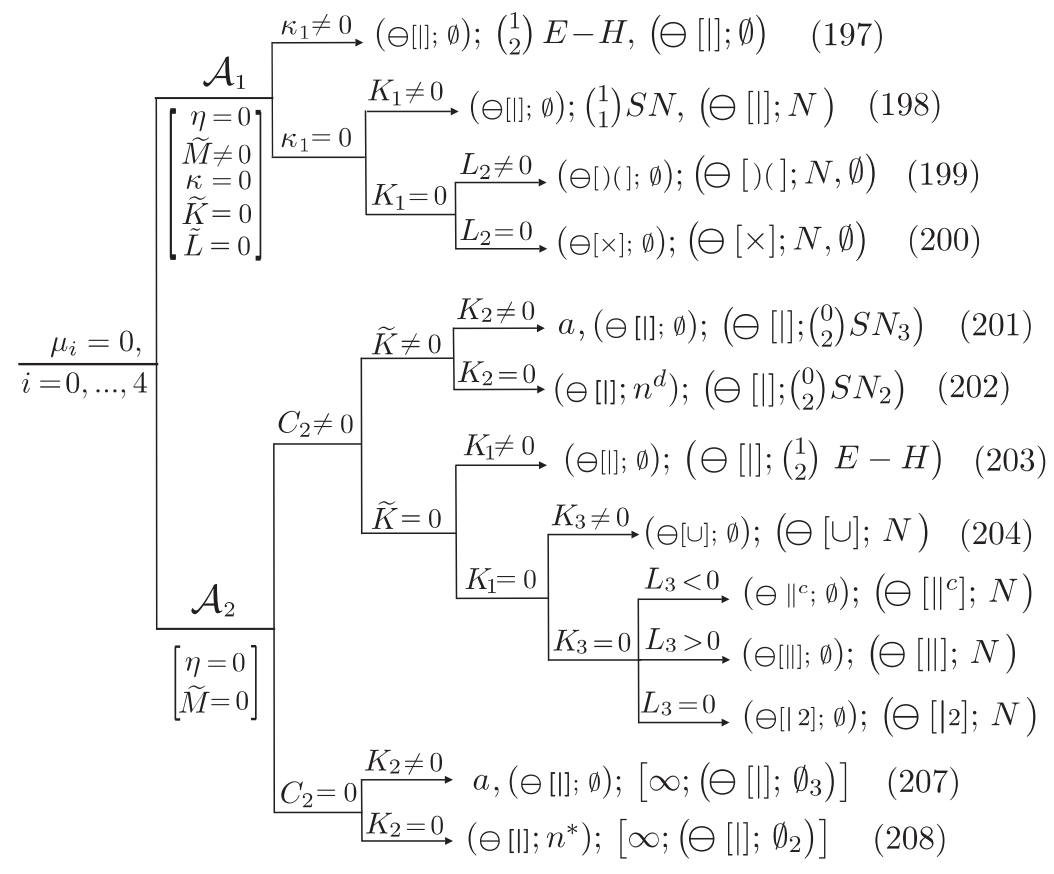

Diagram 6 (cont.): Bifurcation Diagram of global topological configurations: case of degenerate systems $\left(\mu_{0}=\mu_{1}=\mu_{2}=\mu_{3}=\mu_{4}=0\right)$.

of the systems within these charts. Following [1] we use the notation $[\infty ; \emptyset],[\infty ; N],\left[\infty ; N^{d}\right],[\infty ; S],[\infty ; C]$, $\left[\infty ;\left(\begin{array}{l}2 \\ 0\end{array}\right) S N\right]$ or $\left[\infty ;\left(\begin{array}{l}3 \\ 0\end{array}\right) E S\right]$ indicating the singularities obtained after removing the line filled with singularities.

Degenerate systems: We will denote with the symbol $\ominus$ the case when the polynomials defining the system have a common factor. The degeneracy can be produced by a common factor of degree one which defines a straight line or a common quadratic factor which defines a conic. Following [1] we will indicate each case by the following symbols:

- $\ominus[l]$ for a real straight line;

- $\ominus[\circ]$ for a real ellipse;

- $\ominus[\subset]$ for a complex ellipse (i.e. an irreducible conic over $\mathbb{R}$ which has only complex points);

- $\ominus[$ ) (] for an hyperbola;

$\bullet \ominus[\cup]$ for a parabola;

- $\ominus[\times]$ for two real straight lines intersecting at a finite point;

- $\ominus[\cdot]$ for two complex straight lines which intersect at a real finite point;

- $\ominus[\|]$ for two real parallel lines;

- $\ominus\left[\|^{c}\right]$ for two complex parallel lines;

- $\ominus[\mid 2]$ for a double real straight line.

It is worth to notice that the degeneracy $\ominus[(\subset]$ implies the non-existence of real singularities, so we have a chordal system whose phase portrait is equivalent to the case when there are four complex finite singularities and one real infinite singularity. Moreover, the degeneracy $\ominus[\cdot]$ implies the existence of one finite real singularity and its local phase portrait is the same as that of a singularity $h h$ which turns out to be topologically equivalent to a cusp. And finally, we point out that degeneracy $\Theta\left[\|^{c}\right]$ produces one real singularity with configuration $H-H$ at infinity.

Moreover, we also want to determine whether after removing the common factor of the polynomials, singular points remain on the curve defined by this common factor. If the reduced system has no finite singularity on this curve, we will use the symbol $\emptyset$ to describe this situation. If some singular points remain we will use the 
corresponding notation of their various kinds. In this situation, the geometrical properties of the singularity that remain after the removal of the degeneracy, may produce topologically different phenomena, even if they are topologically equivalent singularities. So, we will need to keep the geometrical information associated to that singularity. Some examples of the way we denote the complete notation are:

- $(\ominus[l] ; \emptyset)$ denotes the presence of a real straight line filled up with singular points such that the reduced system has no singularity on this line;

- $(\ominus[\mid] ; f)$ denotes the presence of the same straight line such that the reduced system has a strong focus on this line;

- $\left(\ominus[l] ; n^{d}\right)$ denotes the presence of the same straight line such that the reduced system has a node $n^{d}$ on this line;

- $(\ominus[\cup] ; \emptyset)$ denotes the presence of a parabola filled up with singularities such that no singular point of the reduced system is situated on this parabola.

Degenerate systems with a non-isolated infinite singular point, which however is isolated on the line at infinity: The existence of a common factor of the polynomials defining the differential system also affects the infinite singular points. We point out that the projective completion of a real affine line filled up with singular points has a point on the line at infinity which will then be also a non-isolated singularity.

There is a detailed description of this notation in [1]. In case that after the removal of the finite degeneracy, a singular point at infinity remains at the same place, we must denote it with all its geometrical properties since they may influence the local topological phase portrait. We give below some examples:

- $N, S,(\ominus[1] ; \emptyset)$ means that the system has at infinity a node, a saddle, and one non-isolated singular point which is part of a real straight line filled up with singularities (other that the line at infinity), and that the reduced linear system has no infinite singular point in that position;

- $S,\left(\ominus[l] ; N^{*}\right)$ means that the system has a saddle at infinity, and one non-isolated singular point which is part of a real straight line filled up with singularities (other that the line at infinity), and that the reduced linear system has a star node in that position;

- $S,(\ominus[)(] ; \emptyset, \emptyset)$ means that the system has a saddle at infinity, and two non-isolated singular points which are part of a hyperbola filled up with singularities, and that the reduced constant system has no singularities in those positions;

- $\left(\ominus[\times] ; N^{*}, \emptyset\right)$ means that the system has two non-isolated singular points at infinity which are part of two real intersecting straight lines filled up with singularities, and that the reduced constant system has a star node in one of those positions and no singularity in the other;

- $S,(\ominus[\circ] ; \emptyset, \emptyset)$ means that the system has a saddle at infinity, and two non-isolated (complex) singular points which are the two points at infinity on the complexification of a (real) ellipse, and the reduced constant system has no singularities in those positions.

- $S,\left(\ominus[l] ; N_{3}^{\infty}\right)$ means that the system has a saddle at infinity, and one non-isolated singular point which is part of a real straight line filled up with singularities (other that the line at infinity), and that the reduced linear system has in that position a node such that none of the eigenvectors of the node coincides with the line of singularities and all the orbits (except one) arriving to the node are tangent to the line at infinity.

Degenerate systems with the line at infinity filled up with singularities: According to [1] there are only two geometrical configurations of this class which are also topologically distinct, and which produce just the two phase portraits given in Figure 1. The notations of configurations of infinite singularities in [1] are $\left[\infty ;\left(\ominus[1] ; \emptyset_{3}\right)\right]$ for picture $(a)$ and $\left[\infty ;\left(\ominus[l] ; \emptyset_{2}\right)\right]$, for picture $(b)$.

On the link http://mat.uab.cat/ artes/articles/notation.pdf we offer a table with the geometrical notations of singularities (from which the topological one can be easily extracted) for an easy access during unlimited time in principle. 


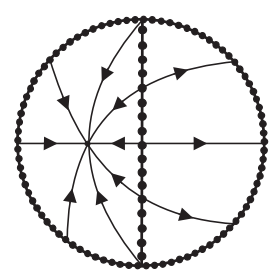

(a)

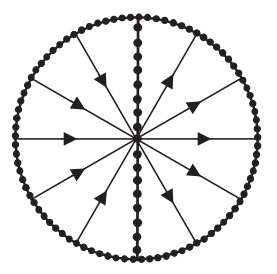

(b)

Figure 1. Phase portraits of quadratic degenerate systems with infinite line filled up with singularities.

\section{INVARIANT POLYNOMIALS ASSOCIATED TO THE GLOBAL TOPOLOGICAL CONFIGURATIONS AND PRELIMINARY RESULTS}

The Diagrams 1-6 classify the global topological configurations of singularities, finite and infinite, of quadratic differential systems yielding 208 such configurations.

For any family of quadratic systems, in whatever normal form, we can then determine from these diagrams the topological global configuration of singularities of the systems in the family. Each one of these configurations is characterized in terms of the following 41 invariant polynomials occurring in Diagrams 1-6:

$$
\begin{gathered}
\left\{\mu_{0}, \ldots, \mu_{4}, \mathbf{D}, \mathbf{P}, \mathbf{R}, \mathbf{S}, \mathbf{T}, \mathbf{U}, E_{1}, E_{3}, \mathcal{T}_{1}, \ldots, \mathcal{T}_{4}, \mathcal{H}, \mathcal{F}, \mathcal{F}_{1}, \ldots, \mathcal{F}_{4}, \mathcal{B}, \mathcal{B}_{1}, \mathcal{B}_{2}, \mathcal{B}_{4}\right. \\
\left.\sigma, \eta, \widetilde{M}, C_{2}, \kappa, \kappa_{1}, \theta_{2}, \theta_{6}, \widetilde{D}, \widetilde{K}, \widetilde{N}, \widetilde{R}, \tilde{L}, K_{1}, K_{2}, K_{3}, L_{1}, L_{2}, L_{3}\right\}
\end{gathered}
$$

Any element of this set could be either an invariant (affine or $G L$-comitant), or a comitant (affine or $G L$ invariant), or a $T$-comitant or a $C T$-comitant (for detailed definitions see [1]).

In what follows we define these elements. We consider the family of quadratic systems:

$$
\begin{aligned}
& \frac{d x}{d t}=p_{0}+p_{1}(x, y)+p_{2}(x, y) \equiv p(x, y), \\
& \frac{d y}{d t}=q_{0}+q_{1}(x, y)+q_{2}(x, y) \equiv q(x, y),
\end{aligned}
$$

where $\max (\operatorname{deg}(p), \operatorname{deg}(q))=2$ and $p_{i}, q_{i}(i=0,1,2)$ are homogeneous polynomials of degree $i$ in $x, y$ in case they are not identically zero:

$$
\begin{array}{lll}
p_{0}=a_{00}, & p_{1}(x, y)=a_{10} x+a_{01} y, & p_{2}(x, y)+a_{20} x^{2}+2 a_{11} x y+a_{02} y^{2}, \\
q_{0}=a_{00}, & q_{1}(x, y)=a_{10} x+a_{01} y, & q_{2}(x, y)+a_{20} x^{2}+2 a_{11} x y+a_{02} y^{2} .
\end{array}
$$

Let $\tilde{a}=\left(a_{00}, a_{10}, a_{01}, a_{20}, a_{11}, a_{02}, b_{00}, b_{10}, b_{01}, b_{20}, b_{11}, b_{02}\right)$ be the 12 -tuple of the coefficients of systems $(3)$ and denote $\mathbb{R}[\tilde{a}, x, y]=\mathbb{R}\left[a_{00}, \ldots, b_{02}, x, y\right]$.

According to [1] (see also [3]) we apply the differential operator $\mathcal{L}=x \cdot \mathbf{L}_{2}-y \cdot \mathbf{L}_{1}$ acting on $\mathbb{R}[\tilde{a}, x, y]$ with

$$
\begin{gathered}
\mathbf{L}_{1}=2 a_{00} \frac{\partial}{\partial a_{10}}+a_{10} \frac{\partial}{\partial a_{20}}+\frac{1}{2} a_{01} \frac{\partial}{\partial a_{11}}+2 b_{00} \frac{\partial}{\partial b_{10}}+b_{10} \frac{\partial}{\partial b_{20}}+\frac{1}{2} b_{01} \frac{\partial}{\partial b_{11}} \\
\mathbf{L}_{2}=2 a_{00} \frac{\partial}{\partial a_{01}}+a_{01} \frac{\partial}{\partial a_{02}}+\frac{1}{2} a_{10} \frac{\partial}{\partial a_{11}}+2 b_{00} \frac{\partial}{\partial b_{01}}+b_{01} \frac{\partial}{\partial b_{02}}+\frac{1}{2} b_{10} \frac{\partial}{\partial b_{11}}
\end{gathered}
$$

to construct several invariant polynomials from the set. More precisely using this operator and the affine invariant $\mu_{0}=\operatorname{Res}_{x}\left(p_{2}(\tilde{a}, x, y), q_{2}(\tilde{a}, x, y)\right) / y^{4}$ we construct the following polynomials

$$
\mu_{i}(\tilde{a}, x, y)=\frac{1}{i !} \mathcal{L}^{(i)}\left(\mu_{0}\right), i=1, . ., 4, \quad \text { where } \mathcal{L}^{(i)}\left(\mu_{0}\right)=\mathcal{L}\left(\mathcal{L}^{(i-1)}\left(\mu_{0}\right)\right)
$$


Using these invariant polynomials we define from the set (2) some new invariants, which according to [1] are responsible for the number and multiplicities of the finite singular points of (3):

$$
\begin{aligned}
& \mathbf{D}=\left[3\left(\left(\mu_{3}, \mu_{3}\right)^{(2)}, \mu_{2}\right)^{(2)}-\left(6 \mu_{0} \mu_{4}-3 \mu_{1} \mu_{3}+\mu_{2}^{2}, \mu_{4}\right)^{(4)}\right] / 48, \\
& \mathbf{P}=12 \mu_{0} \mu_{4}-3 \mu_{1} \mu_{3}+\mu_{2}^{2} \\
& \mathbf{R}=3 \mu_{1}^{2}-8 \mu_{0} \mu_{2} \\
& \mathbf{S}=\mathbf{R}^{2}-16 \mu_{0}^{2} \mathbf{P} \\
& \mathbf{T}=18 \mu_{0}^{2}\left(3 \mu_{3}^{2}-8 \mu_{2} \mu_{4}\right)+2 \mu_{0}\left(2 \mu_{2}^{3}-9 \mu_{1} \mu_{2} \mu_{3}+27 \mu_{1}^{2} \mu_{4}\right)-\mathbf{P R}, \\
& \mathbf{U}=\mu_{3}^{2}-4 \mu_{2} \mu_{4} .
\end{aligned}
$$

In what follows we also need the so-called transvectant of order $k$ (see [7], [8]) of two polynomials $f, g \in$ $\mathbb{R}[\tilde{a}, x, y]$

$$
(f, g)^{(k)}=\sum_{h=0}^{k}(-1)^{h}\left(\begin{array}{l}
k \\
h
\end{array}\right) \frac{\partial^{k} f}{\partial x^{k-h} \partial y^{h}} \frac{\partial^{k} g}{\partial x^{h} \partial y^{k-h}} .
$$

Next we construct the elements $\mathcal{T}_{1}, \ldots, \mathcal{T}_{4}$ of the set $(2)$ which are responsible for the number of the vanishing traces corresponding to the finite singularities of systems (3). For this we define a polynomial (which we call trace polynomial) as follows.

Following to [1] we denote $\sigma(\tilde{a}, x, y)=\frac{\partial p}{\partial x}+\frac{\partial q}{\partial y}=\sigma_{0}(\tilde{a})+\sigma_{1}(\tilde{a}, x, y)$ and we observe that the polynomial $\sigma(\tilde{a}, x, y)$ is an affine comitant of systems $(3)$.

Definition 3 ([11]). We call trace polynomial $\mathfrak{T}(w)$ over the ring $\mathbb{R}[\tilde{a}]$ the polynomial defined as follows

$$
\mathfrak{T}(w)=\sum_{i=0}^{4} \frac{1}{(i !)^{2}}\left(\sigma_{1}^{i}, \frac{1}{i !} \mathcal{L}^{(i)}\left(\mu_{0}\right)\right)^{(i)} w^{4-i}=\sum_{i=0}^{4} \mathcal{G}_{i}(\tilde{a}) w^{4-i}
$$

where the coefficients $\mathcal{G}_{i}(\tilde{a})=\frac{1}{(i !)^{2}}\left(\sigma_{1}^{i}, \mu_{i}\right)^{(i)} \in \mathbb{R}[\tilde{a}], \quad i=0,1,2,3,4\left(\mathcal{G}_{0}(\tilde{a}) \equiv \mu_{0}(\tilde{a})\right)$ are $G L$-invariants.

Using the polynomial $\mathfrak{T}(w)$ we could construct the above mentioned four affine invariants $\mathcal{T}_{4}, \mathcal{T}_{3}, \mathcal{T}_{2}, \mathcal{T}_{1}$ :

$$
\mathcal{T}_{4-i}(\tilde{a})=\left.\frac{1}{i !} \frac{d^{i} \mathfrak{T}}{d w^{i}}\right|_{w=\sigma_{0}} \in \mathbb{R}[\tilde{a}], \quad i=0,1,2,3 \quad\left(\mathcal{T}_{4} \equiv \mathfrak{T}\left(\sigma_{0}\right)\right)
$$

In order to construct the remaining invariant polynomials contained in the set (2) we first need to define some elementary bricks which help us to construct these elements of the set.

We remark that the following polynomials in $\mathbb{R}[\tilde{a}, x, y]$ are the simplest invariant polynomials of degree one with respect to the coefficients of the differential systems (3) which are $G L$-comitants:

$$
C_{i}(x, y)=y p_{i}(x, y)-x q_{i}(x, y), i=0,1,2 ; \quad D_{i}(x, y)=\frac{\partial}{\partial x} p_{i}(x, y)+\frac{\partial}{\partial y} q_{i}(x, y), i=1,2 .
$$

Apart from these simple invariant polynomials we shall also make use of other nine $G L$-invariant polynomials all of which helped in the construction of needed invariant polynomials. The $G L$-invariants are the following:

$$
\begin{aligned}
& T_{1}=\left(C_{0}, C_{1}\right)^{(1)}, \quad T_{2}=\left(C_{0}, C_{2}\right)^{(1)}, \quad T_{3}=\left(C_{0}, D_{2}\right)^{(1)}, \quad T_{4}=\left(C_{1}, C_{1}\right)^{(2)}, \quad T_{5}=\left(C_{1}, C_{2}\right)^{(1)}, \\
& T_{6}=\left(C_{1}, C_{2}\right)^{(2)}, \quad T_{7}=\left(C_{1}, D_{2}\right)^{(1)}, \quad T_{8}=\left(C_{2}, C_{2}\right)^{(2)}, \quad T_{9}=\left(C_{2}, D_{2}\right)^{(1)} \text {. }
\end{aligned}
$$

These are of degree two with respect to the coefficients of systems (3). 
We next define a list of $T$-comitants:

$$
\begin{aligned}
\hat{A}(\tilde{a}) & =\left(C_{1}, T_{8}-2 T_{9}+D_{2}^{2}\right)^{(2)} / 144 \\
\widehat{B}(\tilde{a}, x, y) & =\left\{16 D_{1}\left(D_{2}, T_{8}\right)^{(1)}\left(3 C_{1} D_{1}-2 C_{0} D_{2}+4 T_{2}\right)+32 C_{0}\left(D_{2}, T_{9}\right)^{(1)}\left(3 D_{1} D_{2}\right.\right. \\
& \left.-5 T_{6}+9 T_{7}\right)+2\left(D_{2}, T_{9}\right)^{(1)}\left(27 C_{1} T_{4}-18 C_{1} D_{1}^{2}-32 D_{1} T_{2}+32\left(C_{0}, T_{5}\right)^{(1)}\right) \\
& +6\left(D_{2}, T_{7}\right)^{(1)}\left[8 C_{0}\left(T_{8}-12 T_{9}\right)-12 C_{1}\left(D_{1} D_{2}+T_{7}\right)+D_{1}\left(26 C_{2} D_{1}+32 T_{5}\right)\right. \\
& \left.+C_{2}\left(9 T_{4}+96 T_{3}\right)\right]+6\left(D_{2}, T_{6}\right)^{(1)}\left[32 C_{0} T_{9}-C_{1}\left(12 T_{7}+52 D_{1} D_{2}\right)\right. \\
& \left.-32 C_{2} D_{1}^{2}\right]+48 D_{2}\left(D_{2}, T_{1}\right)^{(1)}\left(2 D_{2}^{2}-T_{8}\right)+6 D_{1} D_{2} T_{4}\left(T_{8}-7 D_{2}^{2}-42 T_{9}\right) \\
& -32 D_{1} T_{8}\left(D_{2}, T_{2}\right)^{(1)}+9 D_{2}^{2} T_{4}\left(T_{6}-2 T_{7}\right)-16 D_{1}\left(C_{2}, T_{8}\right)^{(1)}\left(D_{1}^{2}+4 T_{3}\right) \\
& +12 D_{1}\left(C_{1}, T_{8}\right)^{(2)}\left(C_{1} D_{2}-2 C_{2} D_{1}\right)+12 D_{1}\left(C_{1}, T_{8}\right)^{(1)}\left(T_{7}+2 D_{1} D_{2}\right) \\
& +96 D_{2}^{2}\left[D_{1}\left(C_{1}, T_{6} t\right)^{(1)}+D_{2}\left(C_{0}, T_{6}\right)^{(1)}\right]-4 D_{1}^{3} D_{2}\left(D_{2}^{2}+3 T_{8}+6 T_{9}\right) \\
& \left.-16 D_{1} D_{2} T_{3}\left(2 D_{2}^{2}+3 T_{8}\right)+6 D_{1}^{2} D_{2}^{2}\left(7 T_{6}+2 T_{7}\right)-252 D_{1} D_{2} T_{4} T_{9}\right\} /\left(2^{8} 3^{3}\right) \\
\widehat{D}(\tilde{a}, x, y) & =\left[2 C_{0}\left(T_{8}-8 T_{9}-2 D_{2}^{2}\right)+C_{1}\left(6 T_{7}-T_{6}\right)-\left(C_{1}, T_{5}\right)^{(1)}-9 D_{1}^{2} C_{2}\right. \\
& \left.+6 D_{1}\left(C_{1} D_{2}-T_{5}\right)\right] / 36 \\
\widehat{E}(\tilde{a}, x, y) & =\left[D_{1}\left(2 T_{9}-T_{8}\right)-3\left(C_{1}, T_{9}\right)^{(1)}-D_{2}\left(3 T_{7}+D_{1} D_{2}\right)\right] / 72 \\
\widehat{F}(\tilde{a}, x, y) & =\left[6 D_{1}^{2}\left(D_{2}^{2}-4 T_{9}\right)+4 D_{1} D_{2}\left(T_{6}+6 T_{7}\right)+48 C_{0}\left(D_{2}, T_{9}\right)^{(1)}-9 D_{2}^{2} T_{4}\right. \\
& +288 D_{1} \widehat{E}-24\left(C_{2}, \widehat{D}\right)^{(2)}+120\left(D_{2}, \widehat{D}\right)^{(1)}-36 C_{1}\left(D_{2}, T_{7}\right)^{(1)} \\
& \left.+8 D_{1}\left(D_{2}, T_{5}\right)^{(1)}\right] / 144, \\
\widehat{H}(\tilde{a}, x, y) & =\left(-T_{8}+8 T_{9}+2 D_{2}^{2}\right) / 72 \\
\widehat{K}, y) & \left(T_{8}+4 T_{9}+4 D_{2}^{2}\right) / 72, \\
&
\end{aligned}
$$

as well as the needed bricks:

$$
\begin{aligned}
& \left.\left.\left.A_{1}(\tilde{a})=\hat{A}, \quad A_{2}(\tilde{a})=\left(C_{2}, \widehat{D}\right)^{(3)} / 12, \quad A_{3}(\tilde{a})=\llbracket C_{2}, D_{2}\right)^{(1)}, D_{2}\right)^{(1)}, D_{2}\right)^{(1)} / 48, \\
& A_{4}(\tilde{a})=(\widehat{H}, \widehat{H})^{(2)}, \quad A_{5}(\tilde{a})=(\widehat{H}, \widehat{K})^{(2)} / 2, \quad A_{6}(\tilde{a})=(\widehat{E}, \widehat{H})^{(2)} / 2, \\
& \left.\left.\left.\left.\left.\left.\left.A_{7}(\tilde{a})=\llbracket C_{2}, \widehat{E}\right)^{(2)}, D_{2}\right)^{(1)} / 8, \quad A_{8}(\tilde{a})=\llbracket \widehat{D}, \widehat{H}\right)^{(2)}, D_{2}\right)^{(1)} / 8, \quad A_{9}(\tilde{a})=\llbracket \widehat{D}, D_{2}\right)^{(1)}, D_{2}\right)^{(1)}, D_{2}\right)^{(1)} / 48, \\
& \left.\left.A_{10}(\tilde{a})=\llbracket \widehat{D}, \widehat{K}\right)^{(2)}, D_{2}\right)^{(1)} / 8, \quad A_{11}(\tilde{a})=(\widehat{F}, \widehat{K})^{(2)} / 4, \quad A_{12}(\tilde{a})=(\widehat{F}, \widehat{H})^{(2)} / 4 \text {, } \\
& \left.\left.A_{14}(\tilde{a})=\left(\widehat{B}, C_{2}\right)^{(3)} / 36, \quad A_{15}(\tilde{a})=(\widehat{E}, \widehat{F})^{(2)} / 4, \quad A_{25}(\tilde{a})=\llbracket \widehat{D}, \widehat{D}\right)^{(2)}, \widehat{E}\right)^{(2)} / 16, \\
& \left.\left.\left.\left.\left.\left.\left.\left.A_{33}(\tilde{a})=\llbracket \widehat{D}, D_{2}\right)^{(1)}, \widehat{F}\right)^{(1)}, D_{2}\right)^{(1)}, D_{2}\right)^{(1)} / 128, \quad A_{34}(\tilde{a})=\llbracket \widehat{D}, \widehat{D}\right)^{(2)}, D_{2}\right)^{(1)}, \widehat{K}\right)^{(1)}, D_{2}\right)^{(1)} / 64 \text {. }
\end{aligned}
$$

In the above list the bracket "[" means a succession of two or up to four parentheses "(" depending on the row it appears.

Now we can construct the remaining invariant polynomials of the set (2):

$$
\begin{aligned}
\mathcal{F}(\tilde{a})= & A_{7}, \\
\mathcal{F}_{1}(\tilde{a})= & A_{2}, \\
\mathcal{F}_{2}(\tilde{a})= & -2 A_{1}^{2} A_{3}+2 A_{5}\left(5 A_{8}+3 A_{9}\right)+A_{3}\left(A_{8}-3 A_{10}+3 A_{11}+A_{12}\right)-A_{4}\left(10 A_{8}-3 A_{9}+5 A_{10}\right. \\
& \left.+5 A_{11}+5 A_{12}\right) \\
\mathcal{F}_{3}(\tilde{a})= & -10 A_{1}^{2} A_{3}+2 A_{5}\left(A_{8}-A_{9}\right)-A_{4}\left(2 A_{8}+A_{9}+A_{10}+A_{11}+A_{12}\right)+A_{3}\left(5 A_{8}+A_{10}-A_{11}+5 A_{12}\right), \\
\mathcal{F}_{4}(\tilde{a})= & 20 A_{1}^{2} A_{2}-A_{2}\left(7 A_{8}-4 A_{9}+A_{10}+A_{11}+7 A_{12}\right)+A_{1}\left(6 A_{14}-22 A_{15}\right)-4 A_{33}+4 A_{34}, \\
\mathcal{H}(\tilde{a})= & -\left(A_{4}+2 A_{5}\right), \\
\mathcal{B}(\tilde{a})= & -\left(3 A_{8}+2 A_{9}+A_{10}+A_{11}+A_{12}\right),
\end{aligned}
$$




$$
\begin{aligned}
& \mathcal{B}_{1}(\tilde{a}, x, y)=\left\{\left(T_{7}, D_{2}\right)^{(1)}\left[12 D_{1} T_{3}+2 D_{1}^{3}+9 D_{1} T_{4}+36\left(T_{1}, D_{2}\right)^{(1)}\right]-2 D_{1}\left(T_{6}, D_{2}\right)^{(1)}\right. \\
& \left.\times\left(D_{1}^{2}+12 T_{3}\right)+D_{1}^{2}\left[D_{1}\left(T_{8}, C_{1}\right)^{(2)}+6\left(\left(T_{6}, C_{1}\right)^{(1)}, D_{2}\right)^{(1)}\right]\right\} / 144, \\
& \mathcal{B}_{2}(\tilde{a}, x, y)=\left\{\left(T_{7}, D_{2}\right)^{(1)}\left[8 T_{3}\left(T_{6}, D_{2}\right)^{(1)}-D_{1}^{2}\left(T_{8}, C_{1}\right)^{(2)}-4 D_{1}\left(\left(T_{6}, C_{1}\right)^{(1)}, D_{2}\right)^{(1)}\right]\right. \\
& \left.+\left[\left(T_{7}, D_{2}\right)^{(1)}\right]^{2}\left(8 T_{3}-3 T_{4}+2 D_{1}^{2}\right)\right\} / 384, \\
& \mathcal{B}_{4}(\tilde{a}, x, y)=D_{1}\left(T_{5}+2 D_{2} C_{1}\right)-3 C_{2}\left(D_{1}^{2}+2 T_{3}\right) \text {. } \\
& \widetilde{K}(\tilde{a}, x, y)=4 \widehat{K} \equiv \operatorname{Jacob}\left(p_{2}(\tilde{a}, x, y), q_{2}(\tilde{a}, x, y)\right) \text {, } \\
& \widetilde{M}(\tilde{a}, x, y)=\left(C_{2}, C_{2}\right)^{(2)} \equiv 2 \operatorname{Hess}\left(C_{2}(\tilde{a}, x, y)\right) \text {; } \\
& \eta(\tilde{a})=(\widetilde{M}, \widetilde{M})^{(2)} / 384 \equiv \operatorname{Discrim}\left(C_{2}(\tilde{a}, x, y)\right) ; \\
& \kappa(\tilde{a})=(\widetilde{M}, \widetilde{K})^{(2)} / 4 \text {; } \\
& \kappa_{1}(\tilde{a})=\left(\widetilde{M}, C_{1}\right)^{(2)} ; \\
& \theta_{2}(\tilde{a})=A_{1} ; \\
& \theta_{6}(\tilde{a}, x, y)=C_{1} T_{8}-2 C_{2} T_{6}, \\
& \widetilde{N}(\tilde{a}, x, y)=\widetilde{K}-4 \widehat{H} \\
& K_{1}(\tilde{a}, x, y)=\left(T_{5}-3 C_{2} D_{1}+2 C_{1} D_{2}\right) / 6 \equiv p_{1} q_{2}-p_{2} q_{1} ; \\
& K_{2}(\tilde{a}, x, y)=4\left(T_{2}, \widetilde{M}-2 \widetilde{K}\right)^{(1)}+3 D_{1}\left(C_{1}, \widetilde{M}-2 \widetilde{K}\right)^{(1)}-(\widetilde{M}-2 \widetilde{K})\left(16 T_{3}-3 T_{4} / 2+3 D_{1}^{2}\right) \text {; } \\
& K_{3}(\tilde{a}, x, y)=C_{2}^{2}\left(4 T_{3}+3 T_{4}\right)+C_{2}\left(3 C_{0} \widetilde{K}-2 C_{1} T_{7}\right)+2 K_{1}\left(3 K_{1}-C_{1} D_{2}\right) ; \\
& L_{1}(\tilde{a}, x, y)=\left(C_{2}, \widetilde{D}\right)^{(2)} \text {; } \\
& L_{2}(\tilde{a}, x, y)=\left(C_{2}, \widetilde{D}\right)^{(1)} ; \\
& L_{3}(\tilde{a}, x, y)=C_{1}^{2}-4 C_{0} C_{2} \text {; } \\
& \widetilde{R}(\tilde{a}, x, y)=\tilde{L}+8 \widetilde{K} \text {. } \\
& \tilde{L}(\tilde{a}, x, y)=4 \widetilde{K}-32 \widehat{H}-\widetilde{M} ; \\
& \widetilde{R}(\tilde{a}, x, y)=\tilde{L}+8 \widetilde{K} \text {. } \\
& E_{1}(\tilde{a})=A_{25}, \\
& E_{3}(\tilde{a})=2 A_{1}^{2}-A_{8}-A_{12}, \\
& \widetilde{D}(\tilde{a}, x, y)=\widehat{D} .
\end{aligned}
$$

The following proposition can easily be obtained using results in [11] or Theorem 6.2 in [1]:

Proposition 1. Consider a non-degenerate quadratic differential system. Then:

(i) this system has one center if and only if one of the following sets of conditions holds

$$
\begin{aligned}
& \left(\mathfrak{C}_{1}\right) \mathcal{T}_{4}=0, \mathcal{T}_{3} \mathcal{F}<0, \mathcal{F}_{1}=\mathcal{F}_{2}=\mathcal{F}_{3} \mathcal{F}_{4}=0 ; \\
& \left(\mathfrak{C}_{2}\right) \mathcal{T}_{4}=\mathcal{T}_{3}=0, \mathcal{T}_{2}>0, \mathcal{B}<0, \mathcal{F}=\mathcal{F}_{1}=0 ; \\
& \left(\mathfrak{C}_{3}\right) \mathcal{T}_{4}=\mathcal{T}_{3}=\mathcal{T}_{2}=\mathcal{T}_{1}=0, \sigma \neq 0, \mathcal{F}_{1}=0, \mathcal{H}<0, \mathcal{B}<0, \mathcal{F}=0 ; \\
& \left(\mathfrak{C}_{4}\right) \mathcal{T}_{4}=\mathcal{T}_{3}=\mathcal{T}_{2}=\mathcal{T}_{1}=0, \sigma \neq 0, \mathcal{F}_{1}=0, \mathcal{H}=\mathcal{B}_{1}=0, \mathcal{B}_{2}<0 \\
& \left(\mathfrak{C}_{5}\right) \sigma=0, \mu_{0}<0, \mathbf{D}<0, \mathbf{R}>0, \mathbf{S}>0 ; \\
& \left(\mathfrak{C}_{6}\right) \sigma=0, \mu_{0}=0, \mathbf{D}<0, \mathbf{R} \neq 0 ; \\
& \left(\mathfrak{C}_{7}\right) \sigma=0, \mu_{0}>0, \mathbf{D}>0 ;
\end{aligned}
$$




$$
\begin{aligned}
\left(\mathfrak{C}_{8}\right) \sigma & =0, \mu_{0}>0, \mathbf{D}=0, \mathbf{T}<0 \\
\left(\mathfrak{C}_{9}\right) \sigma & =0, \mu_{0}=\mu_{1}=0, \mu_{2} \neq 0, \mathbf{U}>0, \widetilde{K}=0 \\
\left(\mathfrak{C}_{10}\right) \sigma & =0, \mu_{0}>0, \mathbf{D}=\mathbf{T}=\mathbf{P}=0, \mathbf{R} \neq 0
\end{aligned}
$$

(ii) and it has two centers if and only if one of the following sets of conditions holds

$$
\begin{aligned}
& \left(\widehat{\mathfrak{C}}_{1}\right) \mathcal{T}_{4}=\mathcal{T}_{3}=0, \mathcal{T}_{2}<0, \mathcal{B}<0, \mathcal{H}<0, \mathcal{F}=\mathcal{F}_{1}=0 \\
& \left(\widehat{\mathfrak{C}}_{2}\right) \sigma=0, \mu_{0}>0, \mathbf{D}<0, \mathbf{R}>0, \mathbf{S}>0 .
\end{aligned}
$$

\section{Proof of the Main Theorem}

Proof: Many geometrical properties of singularities which where studied in [1] produce topologically equivalent phenomena. We enumerate them here one by one:

- All types of nodes are locally topologically equivalent among them, and also to foci. We will call all of them anti-saddles. We will keep the name of nodes at infinity since there no focus may exist.

- As already mentioned before, the order of weak singularities associated to saddles and foci is superfluous when we are just interested in the topological configuration of singularities.

- Similarly, information on the types of centers such as isochronous and uniform isochronous centers, is superfluous when we are only interested in topological configuration of singularities.

- Semi-elemental nodes, and saddles are topologically equivalent to elemental nodes and saddles respectively. Moreover, finite semi-elemental saddle-nodes of multiplicity higher than 2 are topologically equivalent to semi-elemental saddle-nodes of multiplicity 2. Even though the multiplicity of singularities can simply be reduced to the minimal value of same parity, we will keep it untouched for some singularities at infinity since the parity must be considered independently for the finite and infinite part of the multiplicities.

- Nilpotent saddles, nodes and saddle-nodes are topologically equivalent to elemental saddles, nodes and semi-elemental saddle-nodes, respectively.

- A nilpotent cusp is topologically equivalent to an intricate singularity $h h$ which is formed by two hyperbolic sectors.

- When studying intricate and nilpotent singularities, the parabolic sectors which appear beside the elliptic sectors are irrelevant when considering local topological equivalence. So, some singularities which are geometrically different will become topologically equivalent to others having less (or even none) parabolic sectors. For example an intricate singularity having only parabolic sectors becomes equivalent with an elementary node.

- Complex singularities (finite or infinite) play no role when considering topological equivalence of singularities, and will simply be removed from the diagrams.

- Degenerate systems, for which we have a common factor $f(x, y)$ of the polynomials $p(x, y)$ and $q(x, y)$ in (1), need special care. In fact, the geometrical configurations of singularities given in [1] yield distinct topological configurations of singularities (and in fact yield even different concrete phase portraits) except in very few cases in which $f(x, y)=0$ is a conic which contains only complex points and thus plays no role in the real phase portrait, obtaining then a previously known configuration. However the cases of two complex conjugate lines (which intersect at a real singularity, finite or infinite) produce interesting phenomena which must be considered with care.

The proof follows by applying the above rules to the Diagrams in [1]. For obtaining the necessary and sufficient conditions in Diagrams 1-6 of this article, we check the conditions in [1] and we discard those which are not necessary for the problem we consider. For example the conditions involving the distinction between a focus and a node, or between weak singularities of various orders (except centers) are not necessary. In some 
particular cases we rewrite some invariant conditions using more adequate invariant polynomials. In the case of centers, the invariant polynomials are grouped in several sets of conditions which are given in Proposition 1.

We distinguish two cases: those with a center and those without.

4.1. The case of the configurations with centers. We must show that each configuration of singularities having a center in the Diagrams from the book [1] has a topological representative in Diagrams 1-6 of this paper and that the corresponding conditions for the existence of a center are equivalent.

4.1.1. Systems with $m_{f}=4$. Here we present a list of all geometrical configurations with centers and finite singularities of total multiplicity four. According to [1] (see Diagrams 8.15, 8.18, 8.20 and 8.21) in the case under consideration we have a total of 30 geometrically distinct configurations of singularities with at least one center. We shall now examine each one of the 30 geometrical configurations one by one.

$\left.\boldsymbol{\alpha}_{1}\right)$ The configuration $s, s, s, c ; N^{f}, N^{f}, N^{f}$ leads to the topological configuration (2). The conditions from the diagram in [1] which define this geometrical configuration are compatible only with the set $\left(\mathfrak{C}_{1}\right)$.

$\boldsymbol{\alpha}_{2}$ ) The configuration $s, s, \$, c ; N^{f}, N^{f}, N^{f}$ leads to the topological configuration (2). The conditions from the diagram in [1] which define this geometrical configuration are compatible only with the set $\left(\mathfrak{C}_{2}\right)$.

$\boldsymbol{\alpha}_{3}$ ) The configuration $\$, \$, \$, c ; N^{f}, N^{f}, N^{f}$ leads to the topological configuration (2). The conditions from the diagram in [1] which define this geometrical configuration are compatible only with the set $\left(\mathfrak{C}_{5}\right)$ because Lemma 8.20 from the book [1] assures us that a system with four distinct real finite singularities having three traces zero must be Hamiltonian, i.e. $\sigma=0$.

$\boldsymbol{\alpha}_{4}$ ) The configuration $s, n, n, c ; S, S, N^{\infty}$ leads to the topological configuration (6). The conditions from the diagram in [1] which define this geometrical configuration are compatible only with the set $\left(\mathfrak{C}_{1}\right)$.

$\boldsymbol{\alpha}_{\mathbf{5}}$ ) The configuration $\$, n, n, c ; S$, (C), (C) leads to the topological configuration (4). The conditions from the diagram in [1] which define this geometrical configuration are compatible only with the set $\left(\mathfrak{C}_{2}\right)$, because the existence of two nodes implies the condition $\mathcal{T}_{2} \neq 0$.

$\boldsymbol{\alpha}_{6}$ ) The configuration $\$, n, n, c ; S, S, N^{\infty}$ leads to the topological configuration (6). The conditions from the diagram in [1] which define this geometrical configuration are compatible only with the set $\left(\mathfrak{C}_{2}\right)$, because the existence of two nodes implies the condition $\mathcal{T}_{2} \neq 0$.

$\left.\boldsymbol{\alpha}_{7}\right)$ The configuration $\$, n, n, c ;\left(\begin{array}{l}0 \\ 3\end{array}\right) S$ leads to the topological configuration (4). The conditions from the diagram in [1] which define this geometrical configuration are compatible only with the set $\left(\mathfrak{C}_{2}\right)$, because the existence of two nodes implies the condition $\mathcal{T}_{2} \neq 0$.

$\boldsymbol{\alpha}_{\mathbf{8}}$ ) The configuration $\$, n^{d}, n^{d}, c ; S$, (C), (c) leads to the topological configuration (4). The conditions from the diagram in [1] which define this geometrical configuration are compatible only with the set $\left(\mathfrak{C}_{2}\right)$, because the existence of two weak singularities implies $\mathcal{T}_{3}=0$, whereas the existence of two nodes implies the condition $\mathcal{T}_{2} \neq 0$.

$\left.\boldsymbol{\alpha}_{\mathbf{9}}\right)$ The configuration $s, s, c, c ; N^{f}$, (C), (C) leads to the topological configuration (9). The conditions from the diagram in [1] which define this geometrical configuration are compatible only with the set $\left(\widehat{\mathfrak{C}}_{1}\right)$.

$\left.\boldsymbol{\alpha}_{10}\right)$ The configuration $\$, \$, c, c ; N^{f}$, (C), (C) leads to the topological configuration (9). The conditions from the diagram in [1] which define this geometrical configuration are compatible only with the set $\left(\widehat{\mathfrak{C}}_{2}\right)$, because by Lemma 8.20 from the book [1] the conditions $\mathcal{T}_{4}=\mathcal{T}_{3}=\mathcal{T}_{2}=0$ imply $\sigma=0$.

$\left.\boldsymbol{\alpha}_{11}\right)$ The configuration $f, c$, , c, (c; $S$, (c) (c) leads to the topological configuration (17). The conditions from the diagram in [1] which define this geometrical configuration are compatible only with the set $\left(\mathfrak{C}_{1}\right)$.

$\left.\boldsymbol{\alpha}_{12}\right)$ The configuration $n, c$, (c), (c) $S, S, N^{\infty}$ leads to the topological configuration (20). The conditions from the diagram in [1] which define this geometrical configuration are compatible only with the set $\left(\mathfrak{C}_{1}\right)$. 
$\left.\boldsymbol{\alpha}_{13}\right)$ The configuration $c, c$, (c), (c); $S$, (c) (c) leads to the topological configuration (18). The conditions from the diagram in [1] which define this geometrical configuration are compatible only with the set $\left(\widehat{\mathfrak{C}}_{1}\right)$ because in this case the condition $\mathcal{T}_{2} \neq 0$ holds (since the complex singularities have non-zero traces).

$\boldsymbol{\alpha}_{\mathbf{1 4 , 1 5}}$ ) The configurations $c, c$, (c), (c) $S, S, N^{\infty}$ and $c^{\odot}, c^{\odot}$, (c), (c); $S, S, N^{\infty}$ lead to the topological configuration (21). The conditions from the diagram in [1] which define these geometrical configurations are compatible only with the set $\left(\widehat{\mathfrak{C}}_{1}\right)$ because in this case the condition $\mathcal{T}_{2} \neq 0$ holds (since the complex singularities have non-zero traces).

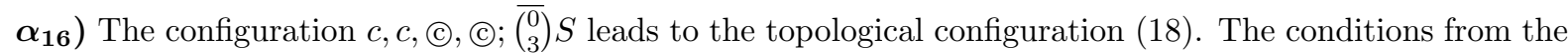
diagram in [1] which define this geometrical configuration are compatible only with the set $\left(\widehat{\mathfrak{C}}_{1}\right)$ because in this case the condition $\mathcal{T}_{2} \neq 0$ holds (since the complex singularities have non-zero traces).

$\boldsymbol{\alpha}_{\mathbf{1 7}, \mathbf{1 8}}$ ) The configurations $c, c$, (C) $^{\tau}$, (C) $^{\tau} ; S$, (C) (c) and $c^{\odot}, c^{\odot}$, (C) ${ }^{\tau},{ }^{\tau}$; $S$, (c) (c) lead to the topological configuration (18). The conditions from the diagram in [1] which define these geometrical configurations are compatible only with the set $\left(\widehat{\mathfrak{C}}_{1}\right)$ because in this case the condition $\mathcal{T}_{2} \neq 0$ holds (since the complex singularities have non-zero traces).

$\left.\boldsymbol{\alpha}_{19}\right)$ The configuration $\$, c$, (c) $^{\rho}$, (c) ${ }^{\rho} N^{f}$, (C), (c) leads to the topological configuration (24). The conditions from the diagram in [1] which define this geometrical configuration are compatible only with the set $\mathfrak{C}_{7}$, because by Lemma 8.28 from the book [1] the conditions $\mathcal{T}_{4}=\mathcal{T}_{3}=\mathcal{T}_{2}=0$ lead to the Hamiltonian systems (i.e. $\sigma=0$ ).

$\boldsymbol{\alpha}_{\mathbf{2 0}, \mathbf{2 1}}$ ) The configurations $\$, c$, (c), (c); $N^{\infty}$, (c), (C) and $\$, c$, (c), (c); $N^{f}$, (c), (c) lead to the topological configuration (24). The conditions from the diagram in [1] which define these geometrical configurations are compatible only with the set $\left(\mathfrak{C}_{2}\right)$.

$\left.\boldsymbol{\alpha}_{\mathbf{2 2 , 2 3}}\right)$ The configurations $\$, c$, (c), (c); $S, N^{\infty}, N^{\infty}$ and $\$, c,\left(\right.$ ), (c); $S, N^{f}, N^{f}$ lead to the topological configuration (26). The conditions from the diagram in [1] which define these geometrical configuratione are compatible only with the set $\left(\mathfrak{C}_{2}\right)$.

$\left.\boldsymbol{\alpha}_{\mathbf{2 4}}\right)$ The configuration $\$, c$, (c), (c); $\left(\begin{array}{l}0 \\ 3\end{array}\right) N$ leads to the topological configuration (24). The conditions from the diagram in [1] which define this geometrical configuration are compatible only with the set $\left(\mathfrak{C}_{2}\right)$.

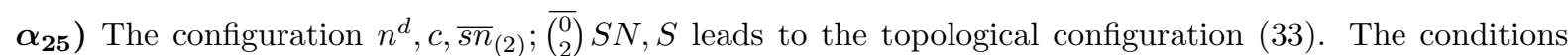
from the diagram in [1] which define this geometrical configuration are compatible only with the set $\left(\mathfrak{C}_{1}\right)$ because in this case the condition $\mathcal{T}_{3} \neq 0$ must hold since there is only one finite singularity with trace zero.

$\boldsymbol{\alpha}_{\mathbf{2 6}}$ ) The configuration $\$, c, \widehat{c p}_{(2)} ; N^{f}$, (C), (c) leads to the topological configuration (41). The conditions from the diagram in [1] which define this geometrical configuration are compatible only with the set $\left(\mathfrak{C}_{8}\right)$ because in this case the condition $\sigma=0$ must hold as it follows from Table $7.1\left(\gamma_{38}\right)$ of [1].

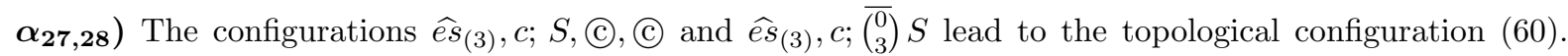
The conditions from the diagram in [1] which define these geometrical configurations are compatible only with the set $\left(\mathfrak{C}_{3}\right)$ because in this case the conditions $\sigma \mathcal{H} \neq 0$ must hold as it follows from Table $7.1\left(\gamma_{23}\right),\left(\gamma_{25}\right)$ of $[1]$.

$\left.\boldsymbol{\alpha}_{29}\right)$ The configuration $\widehat{e s}_{(3)}, c ; S, S, N^{\infty}$ leads to the topological configuration (62). The conditions from the diagram in [1] which define this geometrical configuration are compatible only with the set $\left(\mathfrak{C}_{3}\right)$ because in this case the conditions $\sigma \mathcal{H} \neq 0$ must hold as it follows from Table $7.1\left(\gamma_{24}\right)$ of [1].

$\left.\boldsymbol{\alpha}_{30}\right)$ The configuration $\widehat{s}_{(3)}, c ; N^{f}$, (C), (C) leads to the topological configuration (24). The conditions from the diagram in [1] which define this geometrical configuration are compatible only with the set $\left(\mathfrak{C}_{7}\right)$ because in this case the condition $\sigma=0$ must hold as it follows from Table $7.1\left(\gamma_{39}\right)$ of [1].

4.1.2. Systems with $m_{f} \leq 3$ or degenerate systems. Next we present a list of all geometrical configurations with centers and finite singularities of total multiplicity at most three or with an infinite number of finite singularities. According to [1] (see Diagrams 8.2, 8.6, 8.11, 8.12 and 8.24) in the case under consideration 
we have a total of 18 geometrically distinct configurations of singularities with at least one center. We shall examine each one of them.

$\left.\boldsymbol{\beta}_{\mathbf{1}}\right)$ The configuration $s, s, c ; \widehat{\left(\begin{array}{c}1 \\ 2\end{array}\right)} \stackrel{\widehat{P}}{\curlywedge}_{\curlywedge} E \widehat{P}_{\curlywedge}-H, N^{f}$ leads to the topological configuration (78). The conditions from the diagram in [1] which define this geometrical configuration are compatible only with the set $\left(\mathfrak{C}_{4}\right)$ as it follows from Table $7.1\left(\gamma_{27}\right)$ of [1].

$\boldsymbol{\beta}_{\mathbf{2}}$ ) The configuration $\$, \$, c ; \widehat{\left(\begin{array}{l}1 \\ 2\end{array}\right)} \widehat{P}_{\curlywedge} E \widehat{P}_{\curlywedge}-H, N^{f}$ leads to the topological configuration (78). The conditions from the diagram in [1] which define this geometrical configuration are compatible only with the set $\left(\mathfrak{C}_{6}\right)$ as it follows from Table $7.1\left(\gamma_{40}\right)$ of [1].

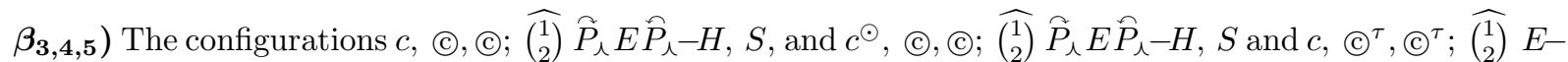
$H, S$ lead to the topological configuration (90). The conditions from the diagram in [1] which define these geometrical configurations are compatible only with the set $\left(\mathfrak{C}_{4}\right)$ as it follows from Table $7.1\left(\gamma_{28 a, b, c}\right)$ of [1].

$\left.\boldsymbol{\beta}_{6}\right)$ The configuration $c$, (c), (c); $\widehat{\left(\begin{array}{l}1 \\ 2\end{array}\right)} \widehat{P}_{\curlywedge} H \widehat{P}_{\curlywedge}-E, S$ leads to the topological configuration (92). The conditions from the diagram in [1] which define this geometrical configuration are compatible only with the set $\left(\mathfrak{C}_{4}\right)$ as it follows from Table $7.1\left(\gamma_{29}\right)$ of [1].

$\left.\boldsymbol{\beta}_{7}\right)$ The configuration $c$, (c), (c); $\widehat{\left(\begin{array}{l}1 \\ 2\end{array}\right)} H_{\curlywedge} H H_{\curlywedge}-H, N^{\infty}$ leads to the topological configuration (94). The conditions from the diagram in [1] which define this geometrical configuration are compatible only with the set $\left(\mathfrak{C}_{4}\right)$ as it follows from Table $7.1\left(\gamma_{30}\right)$ of [1].

$\left.\boldsymbol{\beta}_{\mathbf{8}}\right)$ The configuration $c^{\oplus}$, (c), (c); [ ; $\left.\emptyset\right]$ leads to the topological configuration (97). The conditions from the diagram in [1] which define this geometrical configuration are compatible only with the set $\left(\mathfrak{C}_{4}\right)$ as it follows from Table $7.1\left(\gamma_{31}\right)$ of $[1]$.

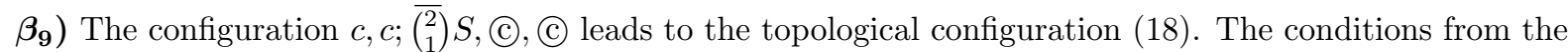
diagram in [1] which define this geometrical configuration are compatible only with the set $\left(\widehat{\mathfrak{C}}_{1}\right)$.

$\boldsymbol{\beta}_{\mathbf{1 0}}$ ) The configuration $\$, c ;\left(\begin{array}{l}2 \\ 1\end{array}\right) N$, (C), (C) leads to the topological configuration (24). The conditions from the diagram in [1] which define this geometrical configuration are compatible only with the set $\left(\mathfrak{C}_{2}\right)$ as it follows from Table $7.1\left(\gamma_{19}\right)$ of $[1]$.

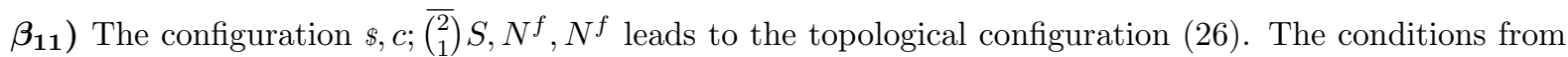
the diagram in [1] which define this geometrical configuration are compatible only with the set $\left(\mathfrak{C}_{2}\right)$ as it follows from Table $7.1\left(\gamma_{21}\right)$ of [1].

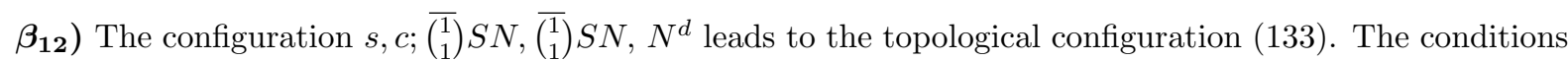
from the diagram in [1] which define this geometrical configuration are compatible only with the set $\left(\mathfrak{C}_{1}\right)$.

$\left.\boldsymbol{\beta}_{13}\right)$ The configuration $\$, c ; N^{d},\left(\begin{array}{l}1 \\ 1\end{array}\right)\left(C,\left(\begin{array}{l}1 \\ 1\end{array}\right)\right.$ C leads to the topological configuration (24). The conditions from the diagram in [1] which define this geometrical configuration are compatible only with the set $\left(\mathfrak{C}_{2}\right)$ as it follows from Table $7.1\left(\gamma_{20}\right)$ of [1].

$\left.\boldsymbol{\beta}_{14}\right)$ The configuration $\$, c ; \overline{\left(\begin{array}{l}1 \\ 1\end{array}\right)} S N, \overline{\left(\begin{array}{l}1 \\ 1\end{array}\right)} S N, N^{d}$ leads to the topological configuration (133). The conditions from the diagram in [1] which define this geometrical configuration are compatible only with the set $\left(\mathfrak{C}_{2}\right)$ as it follows from Table $7.1\left(\gamma_{22}\right)$ of [1].

$\left.\boldsymbol{\beta}_{15}\right)$ The configuration $\$, c ; \widehat{\left(\begin{array}{c}2 \\ 3\end{array}\right)} \widehat{P}_{\curlywedge} \widehat{P}-\widehat{P_{\curlywedge}} \overparen{P}$ leads to the topological configuration (24). The conditions from the diagram in [1] which define this geometrical configuration are compatible only with the set $\left(\mathfrak{C}_{9}\right)$ as it follows from Table $7.1\left(\gamma_{41}\right)$ of [1].

$\left.\boldsymbol{\beta}_{16}\right)$ The configuration $c ; \widehat{\left(\begin{array}{l}3 \\ 2\end{array}\right)} H_{\curlywedge} H H_{\curlywedge}-H, N^{d}$ leads to the topological configuration (94). The conditions from the diagram in [1] which define this geometrical configuration are compatible only with the set $\left(\mathfrak{C}_{4}\right)$ as it follows from Table $7.1\left(\gamma_{32}\right)$ of [1]. 
$\boldsymbol{\beta}_{17}$ ) The configuration $c ; \widehat{\left(\begin{array}{l}1 \\ 2\end{array}\right)} \widetilde{P}_{\curlywedge} E \widehat{P}_{\curlywedge}-H,\left(\begin{array}{l}2 \\ 1\end{array}\right) S$ leads to the topological configuration (90). The conditions from the diagram in [1] which define this geometrical configuration are compatible only with the set $\left(\mathfrak{C}_{4}\right)$ as it follows from Table $7.1\left(\gamma_{33}\right)$ of [1].

$\left.\boldsymbol{\beta}_{18}\right)$ The configuration $c,(\ominus[1] ; \emptyset)$; (C), (C), $(\ominus[l] ; \emptyset)$ leads to the topological configuration (174). The conditions from the diagram in [1] which define this geometrical configuration are compatible only with the set $\left(\mathfrak{C}_{4}\right)$ as it follows from Table $7.1\left(\gamma_{34}\right)$ of $[1]$.

4.2. The case of the configurations without centers. All the topological invariant conditions for configurations of singularities with $m_{f}=4\left(\mu_{0} \neq 0\right)$, appearing in the Diagram 1, can be directly extracted from the geometrical ones, except those of the class with one triple singularity and one simple whose specific configurations are given in Diagram 8.15 of [1]. The main point of this case is to distinguish between a finite semi-elemental triple singularity and a nilpotent triple one with index +1 since the first is a topological node, and the second is an elliptic-saddle. In the case when the triple singularity has index -1 the distinction is not needed since semi-elemental saddles and nilpotent ones are already topologically equivalent. So, an alternative way to distinguish between a semi-elemental triple singularity and a nilpotent one is via the number of traces of singularities that vanish (taking into account the multiplicity of the singularities). If only one trace vanishes, the triple singularity cannot be nilpotent. If two or more vanish, the triple singularity must be nilpotent. From the diagrams of geometrical configurations in [1] it follows that an elliptic-saddle appears only when $E_{3}<0$. So, inside this case, we will use the invariant $\mathcal{T}_{4}^{2}+\mathcal{T}_{3}^{2}$ to decide, when this is zero or not zero, if it is nilpotent or semi-elemental respectively.

In the class of systems with $\mu_{0}=0$ having a simple and a double finite singularities, the key step is to distinguish by means of invariant polynomials between a semi-elemental saddle-node and a nilpotent cusp. And this is simply done by the invariant $E_{1}$ being different from zero or zero, respectively. So we arrive to the conditions of Diagram 2 corresponding to this class.

All other cases can be easily extracted from the diagrams of [1] which give the geometrical configurations leading to the topological ones presented here in Diagrams 1-6.

\section{REFERENCES}

[1] J. C. Artés, J. Llibre and D. Schlomiuk, N. Vulpe, Geometric configurations of singularities of planar polynomial differential systems [A global classification in the quadratic case]. To be published by Birkhäuser, 2019.

[2] J. C. Artés, J. Llibre and N. I. Vulpe, Singular points of quadratic systems: A complete classification in the coefficient space $\mathbb{R}^{12}$. International J. of Bifurcation and Chaos 18 (2008), 313-362.

[3] V. A. Baltag and N. I. Vulpe, Affine-invariant conditions for determining the number and multiplicity of singular points of quadratic differential systems. Izv. Akad. Nauk Respub. Moldova Mat., 1 (1993), 39-48.

[4] C. Chicone and Tian Jinghuang On General Properties of Quadratic Systems. The American Mathematical Monthly, Vol. 89, No. 3 (Mar., 1982), pp. 167-178.

[5] W.A. Coppel A survey of quadratic systems J. Differential Equations21966293-304.

[6] F. Dumortier, J. Llibre and J. C. Artés, Qualitative Theory of Planar Differential Systems. Universitext, Springer-Verlag, New York-Berlin, ISBN: 3-540-32893-9, 2008.

[7] J. H. Grace and A. Young, The algebra of invariants. Stechert, New York, 1941.

[8] P. J. Olver, Classical Invariant Theory. London Math. Soc. Student Texts 44, Cambridge University Press, 1999.

[9] J. Reyn, Phase portraits of planar quadratic systems. Mathematics and Its Applications (Springer), 583. Springer, New York, 2007. xvi+334 pp.

[10] D. Schlomiuk and N. I. Vulpe, The full study of planar quadratic differential systems possessing a line of singularities at infinity. J. Dynam. Differential Equations, 20 (2008), 737-775.

[11] N. Vulpe, Characterization of the finite weak singularities of quadratic systems via invariant theory. Nonlinear Analysis, 74 (2011), No. 4, 6553-6582.

${ }^{1}$ Departament de Matemàtiques, Universitat Autònoma de Barcelona, 08193 Bellaterra, Barcelona, Spain

Email address: artes@mat.uab.cat, jllibre@mat.uab.cat 
2 Département de Mathématiques et de Statistiques, Université de Montréal

Email address: dasch@dms.umontreal.ca

3 Institute of Mathematics And Computer Science, 5 Academiei str, Chişinău, MD-2028, Moldova

Email address: nvulpe@gmail.com 